\title{
Three-Dimensional Modeling of Tsunami Generation and Propagation under the Effect of Stochastic Seismic Fault Source Model in Linearized Shallow-Water Wave Theory
}

\author{
Allam A. Allam, M. A. Omar, and Khaled T. Ramadan \\ Department of Basic and Applied Science, College of Engineering and Technology, Arab Academy for Science, \\ Technology and Maritime Transport, P.O. Box 1029, Abu Quir Campus, Alexandria, Egypt \\ Correspondence should be addressed to M. A. Omar; mabd_elhamid@aast.edu
}

Received 12 September 2013; Accepted 27 November 2013; Published 19 March 2014

Academic Editors: Z. Hou, J. Shen, H. C. So, and X. Wen

Copyright (C) 2014 Allam A. Allam et al. This is an open access article distributed under the Creative Commons Attribution License, which permits unrestricted use, distribution, and reproduction in any medium, provided the original work is properly cited.

\begin{abstract}
Tsunami generation and propagation caused by stochastic seismic fault driven by two Gaussian white noises in the $x$ - and $y$ directions are investigated. This model is used to study the tsunami amplitude amplification under the effect of the noise intensities, spreading uplift length and rise times of the three-dimensional stochastic fault source model. Tsunami waveforms within the frame of the linearized shallow-water theory for constant water depth are analyzed analytically by transform methods (Laplace in time and Fourier in space). The amplification of tsunami amplitudes builds up progressively as time increases during the generation process due to wave focusing while the maximum wave amplitude decreases with time during the propagation process due to the geometric spreading and also due to dispersion. The maximum amplitude amplification is proportional to the propagation length of the stochastic source model and inversely proportional to the water depth. The increase of the normalized noise intensities on the bottom topography leads to an increase in oscillations and amplitude in the free surface elevation. We derived and analyzed the mean and variance of the random tsunami waves as a function of the propagated uplift length, noise intensities, and the average depth of the ocean along the generation and propagation path.
\end{abstract}

\section{Introduction}

A tsunami is most often triggered by undersea earthquakes that cause massive changes to the ocean floor. Tsunami can also be caused by other undersea events such as volcanoes or landslides. When an undersea earthquake or another major disturbance causes a section of the ocean floor to suddenly rise or sink, the mass of water above the affected area also rises or sinks. This unexpected movement of the water creates a series of powerful waves. Undersea earthquakes that cause massive changes to the ocean floor and the displacement of a large volume of water are the most common cause of a tsunami. In recent years, there have been a number of subduction zone earthquakes that have generated unexpectedly large local tsunamis, for example, Papua New Guinea tsunami, 17 July, 1998, Sumatra earthquake and tsunami, 26 December, 2004, Solomon Islands tsunami, 2 April, 2007, Samoa tsunami, 29 September, 2009, the Chile tsunami,
27 February, 2010, and the Fukushima Prefecture, Japan, 11 March, 2011. The investigation of the tsunami waves has become of great practical interest that attracts nowadays a lot of attention due in part to the intensive human activity in coastal areas. The evaluation of a local tsunami threat is useful to get a more effective measure for tsunami warning systems and for protection works. The massive destruction and loss of life associated with the recent tsunamis has underscored the need to develop and implement tsunami hazard mitigation measures. In recent years, significant advances have been made in developing mathematical models to describe the entire process of tsunami event generated by seismic seafloor deformation caused by an underwater earthquake; see [1-3]. Numerical models based on the nondispersive shallow-water equations are often used to simulate tsunami propagation and run-up (e.g., $[4,5])$. The propagation of these waves is strongly influenced by the shape of the bottom. The problem of tsunami source reconstruction is a key concern of tsunami 
research. Certainly, the best way to solve this problem is to measure tsunami waves in the open ocean near to the source area and far away from the distorting influence of the nearshore topography. The sea bottom deformation following an underwater earthquake is a complex phenomenon. This is why, for theoretical or experimental studies, researchers have often used uniform bottom motions such as the vertical motion of a box. Most investigations of tsunami generation and propagation used integral solution (in space and time) for an arbitrary bed displacement based on a linearized description of wave motion in either a two- or three-dimensional fluid domain of uniform depth. The complexity of the integral solutions developed from the linear theory even for the simplest model of bed deformation prevented many authors from determining detailed wave behavior, especially near the source region. In reality the sea bottom deformations following an underwater earthquake are characterized by some rugosity. In practice, the available data about the geometries of the source model are always subject to some uncertainties. The missing information can be modeled by the inclusion of random effects. These circumstances have led several authors to consider water wave propagation in random media; see [6-8]. In this paper, a stochastic model is proposed to describe the bottom irregularity and its effect on the generation and propagation of the tsunami waves. Moreover, the tsunami wave resulting from the random bottom source model is compared with the tsunami wave due to simplified uniform bottom topography in the form of sliding Heaviside step function. Using a stochastic source model, it is demonstrated that local tsunami amplitudes vary by as much as a factor of two or more, depending on the local bathymetry. If another earthquake source parameter such as focal depth is varied in addition to the slip distribution patterns, even greater uncertainty in local tsunami amplitude is expected.

Most previous research has modeled tsunami waves under the effect of deterministic source model. Hammack [9] studied experimentally the generated waves by raising or lowering a box at one end of a channel. He considered two types of time histories: an exponential and a half-sine bed movement. Todorovska and Trifunac [10] investigated the generation and propagation of waves by a slowly spreading uplift of the bottom in linearized shallow-water wave theory. They showed that the effects of the spreading of the ocean floor deformation (faulting, submarine slides, or slumps) on the amplitudes and periods of the generated tsunamis are the largest when the spreading velocity of uplift and the tsunami velocity are comparable. Todorovska et al. [11] investigated tsunami generation by a slowly spreading uplift of the sea floor in the near field considering the effects of the source finiteness and directivity. They described mathematically various two-dimensional kinematic models of submarine slumps and slides as combinations of spreading constant or slopping uplift functions. Their results show that, for given constant water depth, the peak amplitude depends on the ratio of the spreading velocity of the sea floor to the long wavelength tsunami velocity; see [12]. Hayir [13] investigated the motion of a submarine block slide with variable velocities and its effects on the near-field tsunami amplitudes. He found that the amplitudes generated by the slide are almost the same as those created by its average velocity. Both Trifunac et al. [12] and Hayir [13] used very simple kinematic source models represented by Heaviside step functions for representing the generation of tsunami. Dutykh et al. [14] studied the generation of long wave through the ocean by a moving bottom. They demonstrated the differences between the classical approach (passive generation) and the active generation under the effect of the bottom motion. Abou-Dina and Hassan [1] constructed a numerical model of tsunami generation and propagation depending on a nonlinear theory under the effect of a variable bed displacement with constant water depth. They considered nonlinearities and omitted the linear effects of frequency dispersion. Dutykh and Dias [15] studied theoretically the generated waves by multiplying the static deformations caused by slip along a fault by various time laws: instantaneous, exponential, trigonometric, and linear. Kervella et al. [16] performed a comparison between three-dimensional linear and nonlinear tsunami generation models. They observed very good agreement from the superposition of the wave profiles computed with the linear and fully nonlinear models. In addition, they found that the nonlinear shallow-water model was not sufficient to model some of the waves generated by a moving bottom because of the presence of frequency dispersion. Moreover, they suggested that for most events the linear theory is sufficient. Hassan [2] discussed the solution of the nonlinear problem of propagation of waves generated in a homogeneous fluid, occupying an infinite channel, by the bounded motion of the bottom. He demonstrated that the predictions of the linear theory are in good agreement with those of the nonlinear theory for sufficiently small amplitude of the bottom's motion. Hassan et al. [17] investigated the tsunami evolution during its generation under the effect of the variable velocities of realistic submarine landslides based on a two-dimensional curvilinear slide model. They described the tsunami generation from submarine gravity mass flows in three stages: the first stage represented by a rapid curvilinear down and uplift faulting with rise time, the second stage represented by a unilaterally propagation in the positive direction to a significant length to produce curvilinear two-dimensional models represented by a depression slump and a displaced accumulation slide model, and the last stage represented by the time variation in the velocity of the accumulation slide (block slide) by using transforms method. Ramadan et al. [18] studied the nature of the tsunami build-up and propagation during and after realistic curvilinear source models represented by a slow uplift faulting and a spreading slip-fault model. They studied the tsunami amplitude amplification as a function of the spreading velocity and rise time. They also analyzed the normalized peak amplitude as a function of the propagated uplift length, width, and the average depth of the ocean. Ramadan et al. [19] investigated the tsunami generation and propagation resulting from a spreading of a seismic faulting in two orthogonal directions in linearized shallow-water wave theory. They found that the normalized peak amplitudes were smaller when the slip-fault spreads in two orthogonal directions than the numerical values for one-dimensional solution because of the interaction of the velocities. 
It is becoming widely recognized that bottom topography is irregular and difficult to predict and its aspects are best revealed through random source models which are more realistic, because sources of tsunamis are generally uncorrelated. Despite this, a few of the analytical and numerical studies considered stochastic source models for the investigation of the generation and propagation of tsunami waves. This is due to the complexity in the mathematical modeling and analysis of the stochastic case compared to the deterministic case. The main complexity in the stochastic case arises from the difficulty in the derivation of the integral solution of the random profile. Probability of tsunami occurrence frequency on the coast of California was evaluated first by Wiegel [20]. Rascón and Villarreal [21] carried out a statistical study to estimate a stochastic possibility of tsunami hit on the Pacific coast of Mexico. Successively, similar studies have been undertaken to know the local property of the tsunami occurrence frequency. Nakamura [22] considered an extended Poisson process in order to get a better fit to the exceedance probability of local tsunami. Geist [23] determined the effect that ruptures complexity, by way of different slip distribution patterns on the local tsunami wave field under the effect of stochastic source model. Geist [24] developed rapid tsunami models to forecast far-field tsunami amplitudes from initial earthquake information using stochastic source model. de Bouard et al. [7] studied the motion of the free surface of a body of fluid over a variable bottom, in a long wave asymptotic regime. They focused on the two-dimensional case, assuming that the bottom of the fluid region can be described by a stationary random process whose variations take place on short length scales and which are decorrelated on the length scale of the long waves. Craig et al. [25] gave a new derivation and an analysis of long wave model equations for the dynamics of the free surface of a body of water over random variable bottom and illustrated several numerical simulations of nonlinear waves. They considered the case where the bottom is random with uniform statistical properties. They described the asymptotic regime of small amplitude long waves, for which the correlation length of the bottom is short compared to the wavelength. Manouzi and Seaïd [26] developed an efficient numerical method for solving stochastic water waves of Wick type driven by white noise using combination of the WienerItô chaos expansion with a Galerkin finite element method. Their method transforms the stochastic shallow-water equations into a system of deterministic shallow-water equations that are solved for each chaos coefficient in the solution expansion. Dutykh et al. [27] estimated the maximum wave run-up height on a random slope plain shore by solving the classical nonlinear shallow-water equations using finite volume schemes. They compared the results of the random bottom model with the more conventional approaches. Omar et al. [28] studied the nature of the tsunami generation and propagation resulting from unilateral spreading in the $x$ direction of a stochastic source model driven by a Gaussian white noise. Ramadan et al. [29] investigated the tsunami generation and propagation caused by stochastic submarine landslides and slumps driven by a Gaussian white noise in the $x$-and $y$-direction. They found that the increasing in the normalized noise intensities increases the oscillations of the free surface elevation and an increase in the difference in the peak amplitudes of the waveforms in the generation process, while in the propagation process, shows more dispersion and oscillation in the propagated free surface elevation.

In this paper, we investigate the tsunami wave in the nearand far-field using the transform methods (Laplace in time and Fourier in space). A linearized solution for constant water depth is derived for a random sea floor uplift represented by a sliding Heaviside step function under the influence of two independent Gaussian white noise processes in the $x$ - and $y$ directions. The appearance of the Gaussian white noise (an irregular stochastic process) in our bottom topography leads to complications in the integrals resulting from the transform methods. These integrals were found to be of a stochastic type, which are not solvable as a Riemann or Lebesgue integral. Stochastic integrals are mainly classified as Itô or Stratonovich integrals; see [30-34]. In our work, we consider the resultant integrals in Itô sense, which can be solved by many numerical methods; see $[31,33,34]$. The objective of this study is to determine the effect of the stochastic bottom topography on the generation and propagation of the tsunami wave form and discuss aspects of tsunami generation that should be considered in developing this model as well as the propagation wave after the formation of the source model has been completed. Due to the stochastic nature of the bottom topography, the free surface elevation is considered to be a stochastic process; thus, for each realization (sample path) of the bottom topography, there exists a corresponding realization for the free surface elevation. Studying just one realization of the bottom topography has no significance and cannot be used to analyze the behavior of all possible solutions. Therefore, we derive the mean and variance of the free surface elevation. Moreover, we study the fluid wave motion above the finite source, with different noise intensities of the stochastic source model and with variable propagation of the ocean floor uplift, for constant spreading velocity. The effects of dispersion due to the random bottom topography on the tsunami waveforms are treated analytically by means of measuring the variance and illustrating it graphically. In addition, we investigate the mean and the variance of the tsunami waveforms in the near-field and far-field as function of the spreading length, noise intensities, and the water depth.

\section{Mathematical Formulation of the Problem}

Consider a three-dimensional fluid domain $\Lambda$ as shown in Figure 1. It is supposed to represent the ocean above the fault area. It is bounded above by the free surface of the ocean $z=\eta(x, y, t)$ and below by the rigid ocean floor $z=-h(x, y)+\zeta(x, y, t)$, where $\eta(x, y, t)$ is the free surface elevation, $h(x, y)$ is the water depth, and $\zeta(x, y, t)$ is the sea floor displacement function. The domain $\Lambda$ is unbounded in the horizontal directions $x$ and $y$ and can be written as $\Lambda=R^{2} \times[-h(x, y)+\zeta(x, y, t), \eta(x, y, t)]$. For simplicity, $h(x, y)$ is assumed to be a constant. Before the earthquake, the fluid is assumed to be at rest; thus the free surface and the solid boundary are defined by $z=0$ and $z=-h$, respectively. Mathematically, these conditions can be written in the form of initial conditions: $\eta(x, y, 0)=\zeta(x, y, 0)=0$. At time 


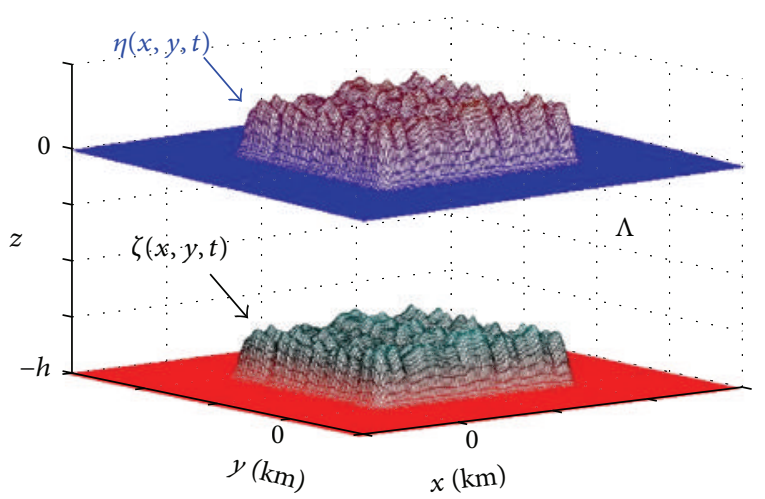

Figure 1: Definition of the fluid domain and coordinate system for a very rapid movement of the assumed random source model.

$t>0$, the bottom boundary moves in a prescribed manner which is given by $z=-h+\zeta(x, y, t)$. The deformation of the sea bottom is assumed to have all the necessary properties needed to compute its Fourier transform in $x$ and $y$ and its Laplace transform in $t$. The resulting deformation of the free surface $z=\eta(x, y, t)$ is to be found as part of the solution. It is assumed that the fluid is incompressible and the flow is irrotational. The former implies the existence of a velocity potential $\Phi(x, y, z, t)$ which fully describes the flow and the physical process. By definition of $\Phi$, the fluid velocity vector can be expressed as $\overrightarrow{\mathbf{q}}=\nabla \Phi$. Thus, the potential flow $\Phi(x, y, z, t)$ must satisfy Laplace's equation

$$
\nabla^{2} \Phi(x, y, z, t)=0 \quad \text { where }(x, y, z) \in \Omega .
$$

The potential $\Phi(x, y, z, t)$ must satisfy the following kinematic and dynamic boundary conditions on the free surface and the solid boundary, respectively:

$$
\begin{gathered}
\Phi_{z}=\eta_{t}+\Phi_{x} \eta_{x}+\Phi_{y} \eta_{y} \quad \text { on } z=\eta(x, y, t), \\
\Phi_{z}=\zeta_{t}+\Phi_{x} \zeta_{x}+\Phi_{y} \zeta_{y} \quad \text { on } z=-h+\zeta(x, y, t), \\
\Phi_{t}+\frac{1}{2}(\nabla \Phi)^{2}+g \eta=0 \quad \text { on } z=\zeta(x, y, t),
\end{gathered}
$$

where $g$ is the acceleration due to gravity. As described above, the initial conditions are given by

$$
\Phi(x, y, z, 0)=\eta(x, y, 0)=\zeta(x, y, 0)=0 .
$$

2.1. Linear Shallow-Water Theory. Various approximations can be considered for the full water wave equations. One is the system of nonlinear shallow-water equations that retains nonlinearity but no dispersion. Solving this problem is a difficult task due to the nonlinearities and the a priori unknown free surface. The concept of shallow water is based on the smallness of the ratio between water depth and wave length. In the case of tsunamis propagating on the surface of deep oceans, one can consider that shallow-water theory is appropriate because the water depth (typically several kilometers) is much smaller than the wave length (typically several hundred kilometers), which is reasonable and usually true for most tsunamis triggered by submarine earthquakes, slumps, and slides; see $[9,15]$. Hence, the problem can be linearized by neglecting the nonlinear terms in the boundary condition (2) and applying the boundary conditions on the nondeformed instead of the deformed boundary surfaces (i.e., $z=-h$ and on $z=0$ instead of $z=-h+\zeta(x, y, t)$ and $z=\eta(x, y, t))$. The linearized problem in dimensional variables can be written as

$$
\nabla^{2} \Phi(x, y, z, t)=0 \quad \text { where }(x, y, z) \in R^{2} \times[-h, 0],
$$

subjected to the following boundary conditions:

$$
\begin{gathered}
\Phi_{z}=\eta_{t} \quad \text { on } z=0, \\
\Phi_{z}=\zeta_{t} \quad \text { on } z=-h, \\
\Phi_{t}+g \eta=0 \quad \text { on } z=0 .
\end{gathered}
$$

The linearized shallow-water solution can be obtained by the Fourier-Laplace transform.

2.2. Solution of the Problem. Our interest is the resulting uplift of the free surface elevation $\eta(x, y, t)$. An analytical analysis is carried out to illustrate the generation of a tsunami for a given bed profile $\zeta(x, y, t)$. Mathematical modeling is carried out to obtain waves generated by vertical and lateral displacements of underwater earthquake using the combined Fourier-Laplace transform of the Laplace equation analytically. All our studies took into account constant depths for which the Laplace and fast Fourier transform (FFT) methods could be applied. Equations (4), (5a), (5b), and (5c) can be solved by using the method of integral transforms. We apply the Fourier transform in $(x, y)$ as follows:

$$
\begin{aligned}
\mathscr{F}_{x y}\{f(x, y)\} & =f^{* *}\left(k_{1}, k_{2}\right) \\
& =\int_{-\infty}^{\infty} \int_{-\infty}^{\infty} e^{-i\left(k_{1} x+k_{2} y\right)} f(x, y) d x d y,
\end{aligned}
$$

with its inverse transform

$$
\begin{aligned}
& \mathscr{F}_{k_{1} k_{2}}^{-1}\left\{f^{* *}\left(k_{1}, k_{2}\right)\right\} \\
& \quad=f(x, y) \\
& \quad=\frac{1}{(2 \pi)^{2}} \int_{-\infty}^{\infty} \int_{-\infty}^{\infty} e^{i\left(x k_{1}+y k_{2}\right)} f^{* *}\left(k_{1}, k_{2}\right) d k_{1} d k_{2},
\end{aligned}
$$

and the Laplace transform in time $t$

$$
\mathscr{L}\{g(t)\}=\widehat{g}(s)=\int_{0}^{\infty} e^{-s t} g(t) d t .
$$

Combining (5a) and (5c) yields the single free surface condition

$$
\Phi_{t t}(x, y, 0, t)+g \Phi_{z}(x, y, 0, t)=0 .
$$


After applying the transforms and using the property $\left[\mathscr{F}\left\{d^{n} f / d x^{n}\right\}=(i k)^{n} f^{*}(k)\right]$, the initial conditions (3), (4), (5b), and (9) become

$$
\begin{gathered}
\widehat{\Phi}_{z z}^{* *}\left(k_{1}, k_{2}, z, s\right)-\left(k_{1}^{2}+k_{2}^{2}\right) \widehat{\Phi}^{* *}\left(k_{1}, k_{2}, z, s\right)=0, \\
\widehat{\Phi}_{z}^{* *}\left(k_{1}, k_{2},-h, s\right)=s \widehat{\zeta}^{* *}\left(k_{1}, k_{2}, s\right), \\
s^{2} \widehat{\Phi}^{* *}\left(k_{1}, k_{2}, 0, s\right)+g \widehat{\Phi}_{z}^{* *}\left(k_{1}, k_{2}, 0, s\right)=0 .
\end{gathered}
$$

The transformed free surface elevation can be obtained from (5c) as

$$
\widehat{\eta}^{* *}\left(k_{1}, k_{2}, s\right)=-\frac{s}{g} \widehat{\Phi}^{* *}\left(k_{1}, k_{2}, 0, s\right)=0
$$

A general solution of (10) will be given by

$$
\begin{aligned}
\widehat{\Phi}^{* *}\left(k_{1}, k_{2}, z, s\right)= & A\left(k_{1}, k_{2}, s\right) \cosh (k z) \\
& +B\left(k_{1}, k_{2}, s\right) \sinh (k z),
\end{aligned}
$$

where $k=\sqrt{k_{1}^{2}+k_{2}^{2}}$. The functions $A\left(k_{1}, k_{2}, s\right)$ and $B\left(k_{1}, k_{2}, s\right)$ can be easily found from the boundary conditions (11) and (12):

$$
\begin{aligned}
& A\left(k_{1}, k_{2}, s\right) \\
& \quad=-\frac{g s \widehat{\zeta}^{* *}\left(k_{1}, k_{2}, s\right)}{\cosh (k h)\left[s^{2}+g k \tanh (k h)\right]}, \\
& B\left(k_{1}, k_{2}, s\right) \\
& \quad=\frac{s^{3} \widehat{\zeta}^{* *}\left(k_{1}, k_{2}, s\right)}{k \cosh (k h)\left[s^{2}+g k \tanh (k h)\right]} .
\end{aligned}
$$

Substituting (15a) and (15b) in the general solution (14) yields

$$
\begin{aligned}
\widehat{\Phi}^{* *}\left(k_{1}, k_{2}, z, s\right)=- & \frac{g s \widehat{\zeta}^{* *}\left(k_{1}, k_{2}, s\right)}{\cosh (k h)\left(s^{2}+\omega^{2}\right)} \\
& \times\left(\cosh (k z)-\frac{s^{2}}{g k} \sinh (k z)\right),
\end{aligned}
$$

where $\omega=\sqrt{g k \tanh (k h)}$ is the circular frequency of the wave motion. The free surface elevation $\eta^{* *}\left(k_{1}, k_{2}, s\right)$ can be obtained from (13) as

$$
\widehat{\eta}^{* *}\left(k_{1}, k_{2}, s\right)=\frac{s^{2} \widehat{\zeta}^{* *}\left(k_{1}, k_{2}, s\right)}{\left(s^{2}+\omega^{2}\right) \cosh (k h)} .
$$

The circular frequency $\omega$ describes the dispersion relation of tsunamis and implies phase velocity $c=\omega / k$ and group velocity $U=d \omega / d k$. Hence, $c=\sqrt{g \tanh (k h) / k}$, and $U=$ $(1 / 2) c(1+2 k h / \sinh (2 k h))$. Since $k=2 \pi / \lambda$, hence as $k h \rightarrow 0$, both $c \rightarrow \sqrt{g h}$ and $U \rightarrow \sqrt{g h}$, which implies that the tsunami velocity $v_{t}=\sqrt{g h}$ for wavelengths $\lambda$ long compared to the water depth $h$. The free surface elevation $\eta(x, y, t)$ can be evaluated for a specified $\zeta(x, y, t)$ by obtaining its transform $\hat{\zeta}^{* *}\left(k_{1}, k_{2}, s\right)$, then substituting it into (17), and inverting $\hat{\eta}^{* *}\left(k_{1}, k_{2}, s\right)$ analytically to obtain $\eta^{* *}\left(k_{1}, k_{2}, t\right)$ which is further converted to $\eta(x, y, t)$ by using double inverse Fourier transform. The above linearized solution is known as the shallow-water solution. We considered the stochastic source model for the sea floor displacement as a variable slip-fault, propagating unilaterally in the positive $x$ direction for time $0 \leq t \leq t^{*}$ with finite velocity $v$. In the $y$-direction, the model randomly propagates instantaneously.

The free surface elevation $\eta^{* *}\left(k_{1}, k_{2}, t\right)$ can be evaluated by using inverse Laplace transform of $\eta^{* *}\left(k_{1}, k_{2}, s\right)$ from the Convolution theorem as follows:

$$
\begin{aligned}
\eta^{* *}\left(k_{1}, k_{2}, t\right)= & \frac{1}{\cosh (k h)} \\
& \times\left[\zeta^{* *}\left(k_{1}, k_{2}, t\right)\right. \\
& \left.\quad-\omega \int_{0}^{t} \sin (\omega(t-u)) \zeta^{* *}\left(k_{1}, k_{2}, u\right) d u\right] .
\end{aligned}
$$

The sea floor displacement is taken as

$$
\zeta(x, t)=\zeta_{1}(x, t)+\zeta_{2}(x, t)
$$

where

$$
\begin{gathered}
\zeta_{1}(x, t)=\zeta_{0} H\left(t-\frac{x}{v}\right), \\
\zeta_{2}(x, t)=\left(\sigma_{x_{0}} \xi(x)+\sigma_{y_{0}} \xi(y)\right) H\left(t-\frac{x}{v}\right),
\end{gathered}
$$

where $H(x)$ represents the Heaviside function, $\zeta_{0}$ denotes the initial uplift of the bottom topography, $\xi_{x}(x)$ and $\xi_{y}(y)$ denote two independent Gaussian white noise processes with two real valued parameters $\sigma_{x_{0}}, \sigma_{y_{0}} \geq 0$ that control the strength of the induced noises in the $x$ - and $y$-directions, respectively, and $v$ is the rapture velocity of the stochastic bottom in the $x$-direction.

Laplace and Fourier transforms can now be applied to the bottom topography (19) to obtain

$$
\widehat{\zeta}^{* *}\left(k_{1}, k_{2}, s\right)=\widehat{\zeta}_{1}^{* *}\left(k_{1}, k_{2}, s\right)+\widehat{\zeta}_{2}^{* *}\left(k_{1}, k_{2}, s\right),
$$

where

$$
\begin{gathered}
\hat{\zeta}_{1}^{* *}\left(k_{1}, k_{2}, s\right)=\frac{1-e^{-i k_{2} D}}{i k_{2}} \frac{\zeta_{0}}{s} \frac{1-e^{-\left(i k_{1}+s / v\right) L}}{i k_{1}+s / v}, \\
\hat{\zeta}_{2}^{* *}\left(k_{1}, k_{2}, s\right) \\
=\frac{\sigma_{x_{0}}}{s} \frac{1-e^{-i k_{2} D}}{i k_{2}} \int_{0}^{L} e^{-\left(i k_{1}+s / v\right) x} \xi(x) d x \\
\quad+\frac{\sigma_{y_{0}}}{s} \frac{1-e^{-\left(i k_{1}+s / v\right) L}}{\left(i k_{1}+s / v\right)} \int_{0}^{D} e^{-i k_{2} y} \xi(y) d y,
\end{gathered}
$$

where $L$ and $D$ are the propagated length and width of the stochastic bottom topography in the $x$-and $y$-direction, respectively. 
The transformed free surface elevation, $\widehat{\eta}^{* *}\left(k_{1}, k_{2}, s\right)$, can be obtained by substituting (22) into (17) as

$$
\widehat{\eta}^{* *}\left(k_{1}, k_{2}, s\right)=\widehat{\eta}_{1}^{* *}\left(k_{1}, k_{2}, s\right)+\widehat{\eta}_{2}^{* *}\left(k_{1}, k_{2}, s\right),
$$

where

$$
\widehat{\eta}_{i}^{* *}\left(k_{1}, k_{2}, s\right)=\frac{s^{2} \widehat{\zeta}_{i}^{* *}\left(k_{1}, k_{2}, s\right)}{\left(s^{2}+\omega^{2}\right) \cosh (k h)} \quad i=1,2 .
$$

Substituting (24) into (26) yields

$$
\begin{aligned}
\widehat{\eta}_{2}^{* *}\left(k_{1}, k_{2}, s\right) & \frac{s}{\left(s^{2}+\omega^{2}\right) \cosh (k h)} \\
& \times\left[\sigma_{x_{0}} \frac{1-e^{-i k_{2} D}}{i k_{2}} \int_{0}^{L} e^{-\left(i k_{1}+s / v\right) x} \xi(x) d x\right. \\
& \left.\quad+\sigma_{y_{0}} \frac{1-e^{-\left(i k_{1}+s / v\right) L}}{\left(i k_{1}+s / v\right)} \int_{0}^{D} e^{-i k_{2} y} \xi(y) d y\right] .
\end{aligned}
$$

The free surface elevation $\eta_{2}^{* *}\left(k_{1}, k_{2}, t\right)$ can be evaluated by using inverse Laplace transform of $\widehat{\eta}_{2}^{* *}\left(k_{1}, k_{2}, s\right)$ as follows:

$$
\begin{aligned}
& \eta_{2}^{* *}\left(k_{1}, k_{2}, t\right) \\
& =\frac{1}{\cosh (k h)} \\
& \times\left[\sigma_{x_{0}} \frac{1-e^{-i k_{2} D}}{i k_{2}} \int_{0}^{L} e^{-i k_{1} x} \cos \left(\omega\left(t-\frac{x}{v}\right)\right)\right. \\
& \times H\left(t-\frac{x}{v}\right) \xi(x) d x \\
& +\sigma_{y_{0}} v \int_{0}^{D} e^{-i k_{2} y} \xi(y) d y \\
& \left.\times \int_{0}^{t} \cos (\omega u) e^{-i k_{1} v(t-u)} H\left(t^{*}-t+u\right) d u\right] \\
& =\frac{1}{\cosh (k h)} \\
& \times\left[\sigma_{x_{0}} \frac{1-e^{-i k_{2} D}}{i k_{2}}\right. \\
& \times\left\{\begin{array}{rr}
\int_{0}^{L} e^{-i k_{1} x} \cos \left(\omega\left(t-\frac{x}{v}\right)\right) & d W(x) \\
t & >t^{*} \\
\int_{0}^{t v} e^{-i k_{1} x} \cos \left(\omega\left(t-\frac{x}{v}\right)\right) & d W(x) \\
t & \leq t^{*}
\end{array}\right.
\end{aligned}
$$

$$
\begin{aligned}
& +\sigma_{y_{0}} v \int_{0}^{D} e^{-i k_{2} y} d W(y) \\
& \times\left\{\begin{array}{c}
\int_{t-t^{*}}^{t} e^{-i k_{1} v(t-u)} \cos (\omega u) d u \\
t>t^{*} \\
\int_{0}^{t} e^{-i k_{1} v(t-u)} \cos (\omega u) d u \\
t \leq t^{*}
\end{array}\right],
\end{aligned}
$$

where $t^{*}=L / v$. The integrals in (28) are stochastic integrals that can be considered as Itô integrals. Such integrals can be solved by various methods; see [31, 34]. The solution $\widehat{\eta}_{1}^{* *}\left(k_{1}, k_{2}, s\right)$ for $\widehat{\zeta}_{1}^{* *}\left(k_{1}, k_{2}, s\right)$ can be obtained from (23) and (26) as

$$
\widehat{\eta}_{1}^{* *}\left(k_{1}, k_{2}, s\right)=\frac{\zeta_{0}}{\cosh (k h)} \frac{1-e^{-i k_{2} D}}{i k_{2}} \frac{v\left(1-e^{-\left(i k_{1} v+s\right) t^{*}}\right)}{i k_{1} v+s},
$$

which can be transformed to the time domain as

$$
\begin{aligned}
& \eta_{1}^{* *}\left(k_{1}, k_{2}, t\right) \\
& =\left(\frac{\zeta_{0}}{\cosh (k h)} \frac{1-e^{-i k_{2} D}}{i k_{2}} \frac{v}{\omega^{2}-k_{1}^{2} v^{2}}\right) \\
& \times\left\{\begin{array}{c}
\omega \sin (w t)+i k_{1} v \cos (w t) \\
-e^{-i k_{1} v t^{*}}\left[\omega \sin \left(w\left(t-t^{*}\right)\right)\right. \\
\left.+i k_{1} v \cos \left(w\left(t-t^{*}\right)\right)\right], \\
\text { for } t>t^{*} \\
\omega \sin (w t)+i k_{1} v \cos (w t) \\
-i k_{1} v e^{-i k_{1} v t}, \quad \text { for } t \leq t^{*} .
\end{array}\right.
\end{aligned}
$$

This completes the solution of the problem, $\eta^{* *}\left(k_{1}, k_{2}\right.$, $t)=\eta_{1}^{* *}\left(k_{1}, k_{2}, t\right)+\eta_{2}^{* *}\left(k_{1}, k_{2}, t\right)$, in the double Fourier transform domain.

Finally, $\eta(x, y, t)$ is evaluated using the inverse fast Fourier transform (IFFT). The IFFT is a fast algorithm for efficient implementation of the inverse discrete Fourier transform (IDFT). In this paper, this inversion is done using the Matlab IFFT algorithm.

In order to implement the algorithm efficiently, singularities should be removed by finite limits as follows.

(a) As $k \rightarrow 0$ implies $k_{1} \rightarrow 0, k_{2} \rightarrow 0$, and $\omega \rightarrow 0$, then $\eta_{1}^{* *}\left(k_{1}, k_{2}, t\right)$ and $\eta_{2}^{* *}\left(k_{1}, k_{2}, t\right)$ have the following limits:

$$
\begin{gathered}
\lim _{k \rightarrow 0} \eta_{1}^{* *}\left(k_{1}, k_{2}, t\right)=\left(\zeta_{0} v D\right) \begin{cases}t^{*}, & \text { for } t>t^{*}, \\
t, & \text { for } t \leq t^{*},\end{cases} \\
\lim _{k \rightarrow 0} \eta_{2}^{* *}\left(k_{1}, k_{2}, t\right)=\sigma_{x_{0}} D \begin{cases}W(L), & \text { for } t>t^{*}, \\
W(t v), & \text { for } t \leq t^{*}\end{cases} \\
+\sigma_{y_{0}} v W(D) \begin{cases}t^{*}, & \text { for } t>t^{*} \\
t, & \text { for } t \leq t^{*} .\end{cases}
\end{gathered}
$$




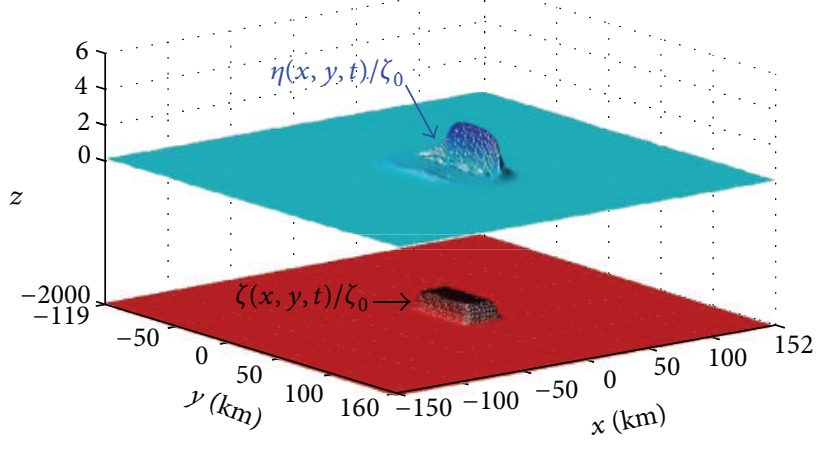

(a) $t=0.25 t^{*}$

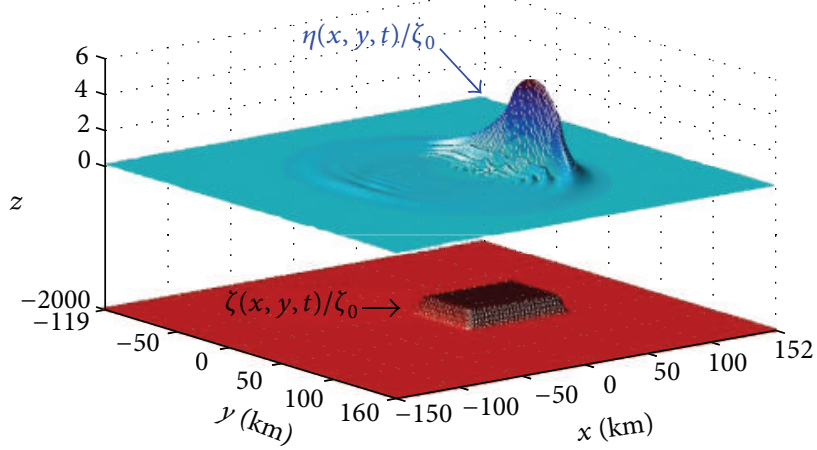

(c) $t=0.75 t^{*}$

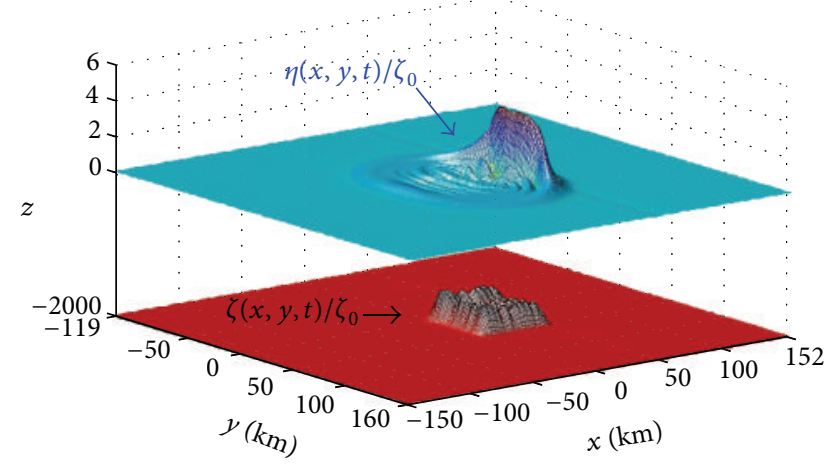

(b) $t=0.5 t^{*}$

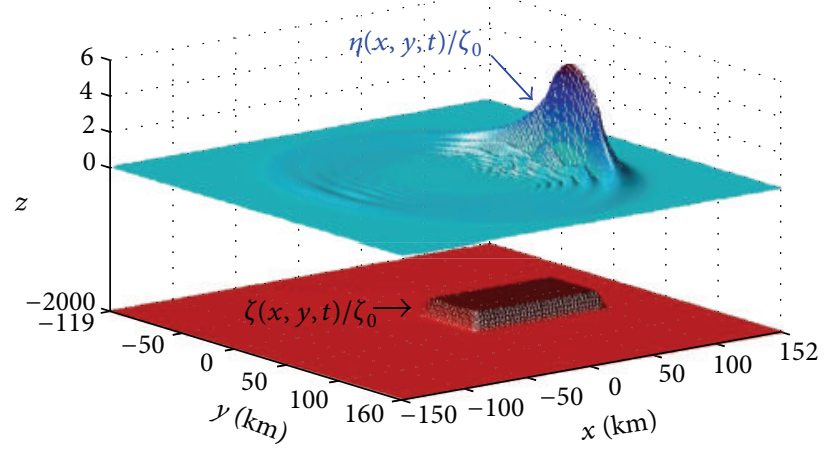

(d) $t=t^{*}$

FiguRE 2: Generation of the normalized tsunami waveforms, $\eta(x, y, t) / \zeta_{0}$, for different rise times at $\sigma_{x}=\sigma_{y}=0, L=100 \mathrm{~km}, D=50 \mathrm{~km}$, $t^{*}=L / v$, and $v=v_{t}$.

(b) As $k_{1} \rightarrow 0$ implies $k=k_{2}$ and $\omega=\sqrt{g k_{2} \tanh \left(k_{2} h\right)}$, then $\eta_{1}^{* *}\left(k_{1}, k_{2}, t\right)$ and $\eta_{2}^{* *}\left(k_{1}, k_{2}, t\right)$ have the following limits:

$$
\begin{aligned}
\lim _{k_{1} \rightarrow 0} \eta_{1}^{* *}\left(k_{1}, k_{2}, t\right) & \\
= & \left(\frac{\zeta_{0}}{\cosh \left(k_{2} h\right)} \frac{1-e^{-i k_{2} D}}{i k_{2}} \frac{v}{\omega}\right) \\
& \times \begin{cases}\sin (w t)-\sin \left(w\left(t-t^{*}\right)\right), & \text { for } t>t^{*}, \\
\sin (w t), & \text { for } t \leq t^{*},\end{cases}
\end{aligned}
$$$$
\lim _{k_{1} \rightarrow 0} \eta_{2}^{* *}\left(k_{1}, k_{2}, t\right)
$$$$
=\frac{1}{\cosh \left(k_{1} h\right)}
$$$$
\times\left[\sigma _ { x _ { 0 } } \frac { 1 - e ^ { - i k _ { 2 } D } } { i k _ { 2 } } \left\{\begin{array}{r}
\int_{0}^{L} \cos \left(\omega\left(t-\frac{x}{v}\right)\right) d W(x) \\
\int_{0}^{t v} \cos \left(\omega\left(t-\frac{x}{v}\right)\right) d W(x) \\
\text { for } t \leq t^{*}
\end{array}\right.\right.
$$

$$
\begin{aligned}
& +\sigma_{y_{0}} v \int_{0}^{D} e^{-i k_{2} y} d W(y) \\
& \times\left\{\begin{array}{ll}
\frac{1}{\omega}\left(\sin (\omega t)-\sin \left(\omega\left(t-t^{*}\right)\right)\right) & \text { for } t>t^{*} \\
\frac{1}{\omega} \sin (\omega t) & \text { for } t \leq t^{*}
\end{array}\right] .
\end{aligned}
$$

(c) As $k_{2} \rightarrow 0$ implies $k=k_{1}$ and $\omega=\sqrt{g k_{1} \tanh \left(k_{1} h\right)}$, then $\eta_{1}^{* *}\left(k_{1}, k_{2}, t\right)$ and $\eta_{2}^{* *}\left(k_{1}, k_{2}, t\right)$ have the following limits:

$$
\begin{aligned}
& \lim _{k_{2} \rightarrow 0} \eta_{1}^{* *}\left(k_{1}, k_{2}, t\right) \\
& =\left(\frac{\zeta_{0}}{\cosh \left(k_{1} h\right)} \frac{v D}{\omega^{2}-k_{1}^{2} v^{2}}\right) \\
& \quad \times\left\{\begin{array}{r}
\omega \sin (w t)+i k_{1} v \cos (w t), \\
-e^{-i k_{1} v t^{*}}\left[\omega \sin \left(w\left(t-t^{*}\right)\right),\right. \\
\left.+i k_{1} v \cos \left(w\left(t-t^{*}\right)\right)\right] \\
\text { for } t>t^{*}, \\
\omega \sin (w t)+i k_{1} v \cos (w t)-i k_{1} v e^{-i k_{1} v t}, \\
\text { for } t \leq t^{*},
\end{array}\right.
\end{aligned}
$$




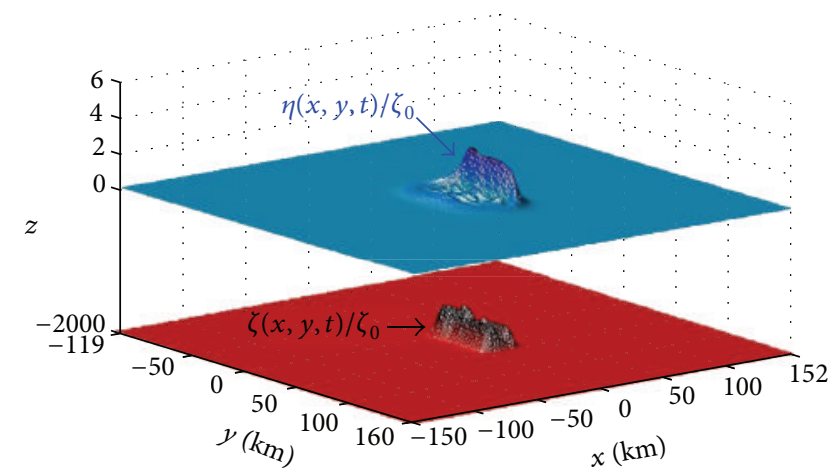

(a) $t=0.25 t^{*}$

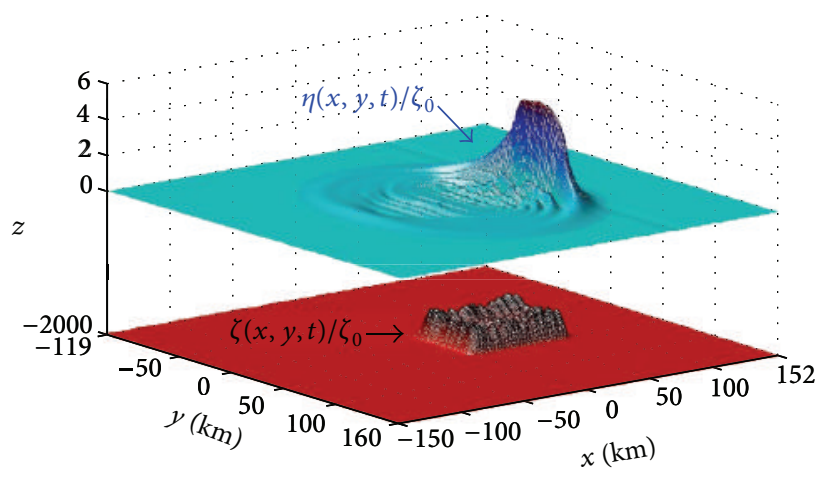

(c) $t=0.75 t^{*}$

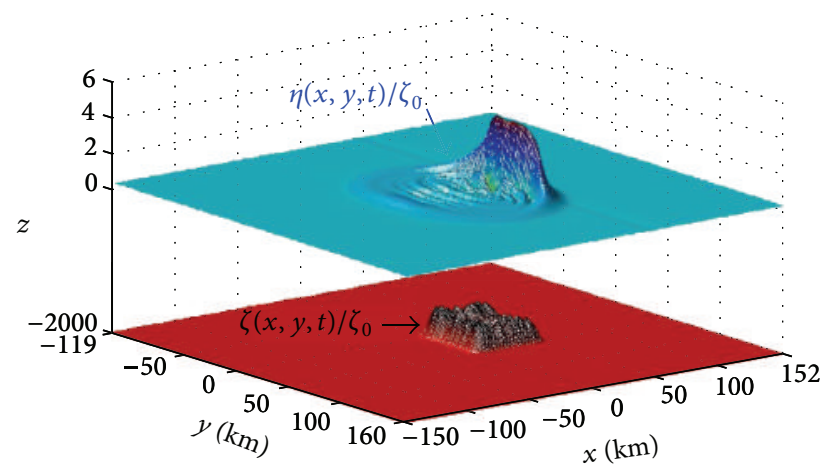

(b) $t=0.5 t^{*}$

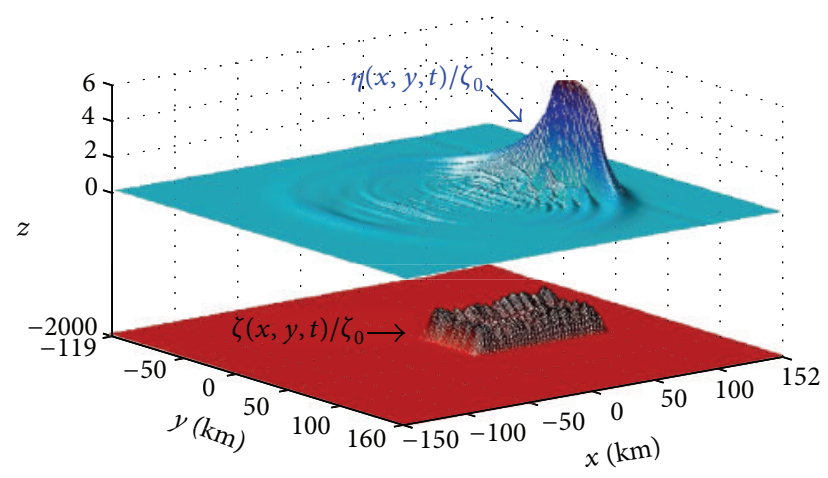

(d) $t=t^{*}$

Figure 3: Generation of the normalized tsunami waveforms, $\eta(x, y, t) / \zeta_{0}$, for a sample trajectory of the stochastic source model at different rise times with $\sigma_{x}=\sigma_{y}=0.2, L=100 \mathrm{~km}, D=50 \mathrm{~km}, t^{*}=L / v$, and $v=v_{t}$.

$$
\begin{aligned}
\lim _{k_{1} \rightarrow 0} \eta_{2}^{* *}\left(k_{1}, k_{2}, t\right) & \frac{1}{\cosh (k h)} \\
\times & {\left[\sigma _ { x _ { 0 } } D \left\{\begin{array}{ll}
\int_{0}^{L} e^{-i k_{1} x} \cos \left(\omega\left(t-\frac{x}{v}\right)\right) d W(x) & t>t^{*}, \\
\int_{0}^{t v} e^{-i k_{1} x} \cos \left(\omega\left(t-\frac{x}{v}\right)\right) d W(x) & t \leq t^{*}
\end{array}\right.\right.} \\
& +\sigma_{y_{0}} v W(D)\left\{\begin{array}{ll}
\int_{t-t^{*}}^{t} e^{-i k_{1} v(t-u)} \cos (\omega u) d u & t>t^{*} \\
\int_{0}^{t} e^{-i k_{1} v(t-u)} \cos (\omega u) d u & t \leq t^{*}
\end{array}\right] .
\end{aligned}
$$

2.3. Mathematical Derivation of the Mean and Variance of the Stochastic Tsunami Waveforms. The presence of Gaussian white noise in the model of the bottom topography leads to randomness in the generated waveforms. These waveforms are considered to be a stochastic process, which has an infinite number of trajectories. All these trajectories are considered as solutions for our stochastic model, but some trajectories may be more probable than others. Thus, instead of dealing with only one of these trajectories, we derive mathematically the mean and variance of the stochastic tsunami waveforms to get a better insight of the overall behavior of the stochastic tsunami waveforms; see [35].

First, we derive the mean of the tsunami waveforms by expressing the transformed free surface elevation, $\widehat{\eta}^{* *}\left(k_{1}, k_{2}, s\right)$, using (27) and (29) as

$$
\begin{aligned}
\widehat{\eta}^{* *}\left(k_{1}, k_{2}, s\right)= & \widehat{\eta}_{1}^{* *}\left(k_{1}, k_{2}, s\right) \\
& +\widehat{\alpha}_{x}^{* *}\left(k_{1}, k_{2}, s\right) \int_{0}^{L} e^{-\left(i k_{1}+s / v\right) x} d W(x) \\
& +\widehat{\alpha}_{y}^{* *}\left(k_{1}, k_{2}, s\right) \int_{0}^{D} e^{-i k_{2} y} d W(y),
\end{aligned}
$$

where

$$
\begin{aligned}
& \widehat{\alpha}_{x}^{* *}\left(k_{1}, k_{2}, s\right)=\frac{\sigma_{x}}{\cosh (k h)} \frac{1-e^{-i k_{2} D}}{i k_{2}} \frac{s}{\left(s^{2}+\omega^{2}\right)} \\
& \widehat{\alpha}_{y}^{* *}\left(k_{1}, k_{2}, s\right)=\frac{\sigma_{y}}{\cosh (k h)} \frac{1-e^{-\left(i k_{1}+s / v\right) L}}{i k_{1}+s / v} \frac{s}{\left(s^{2}+\omega^{2}\right)} .
\end{aligned}
$$

Taking the expectation of (34) and using the property that the expectation of an Itô integral equals zero, see [32], yield

$$
E\left[\widehat{\eta}^{* *}\left(k_{1}, k_{2}, s\right)\right]=\widehat{\eta}_{1}^{* *}\left(k_{1}, k_{2}, s\right) .
$$

As the free surface elevation is a continuous function in $(x, y, t)$, thus Fubini's theorem, see page 64 in Kloeden and 


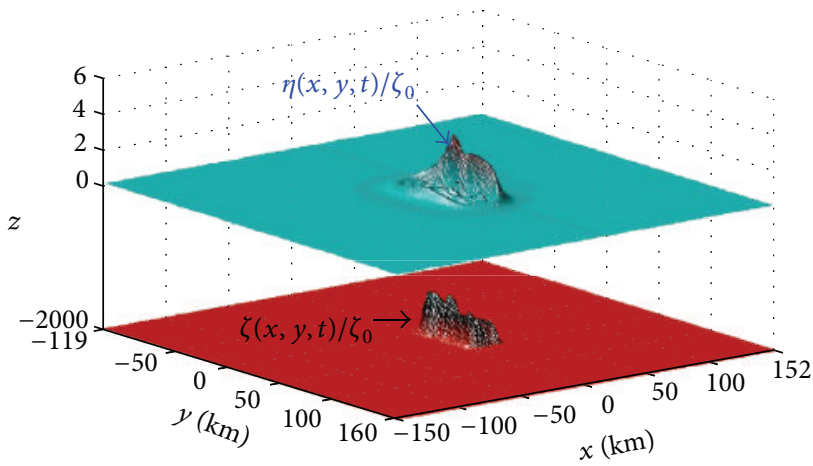

(a) $t=0.25 t^{*}$

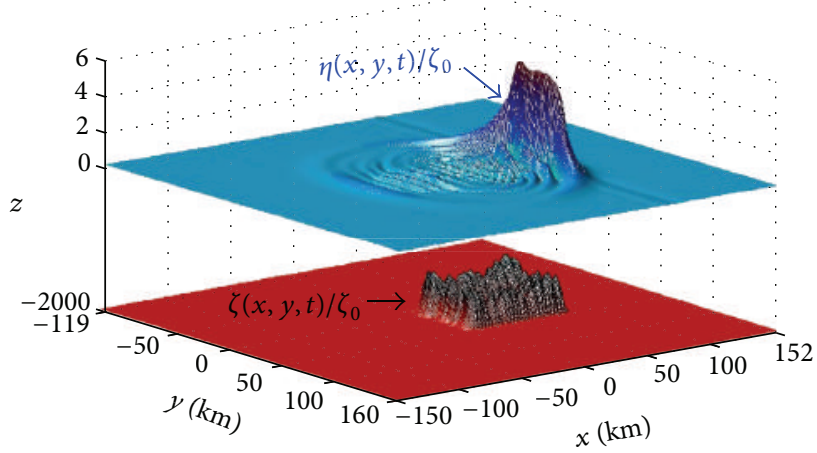

(c) $t=0.75 t^{*}$

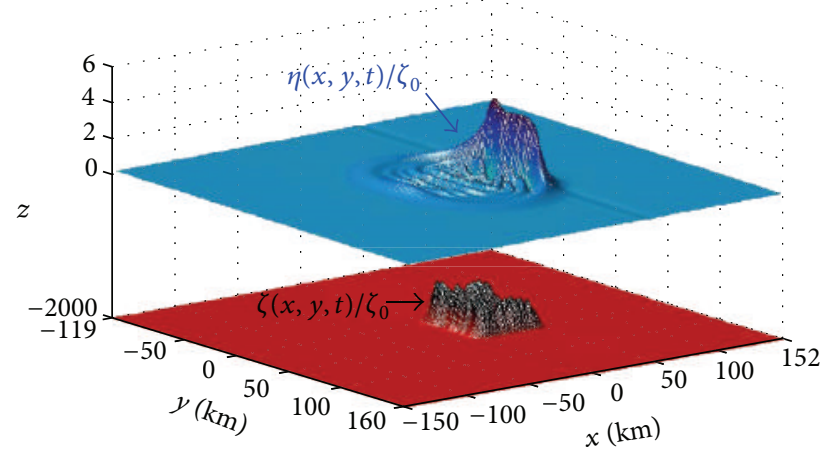

(b) $t=0.5 t^{*}$

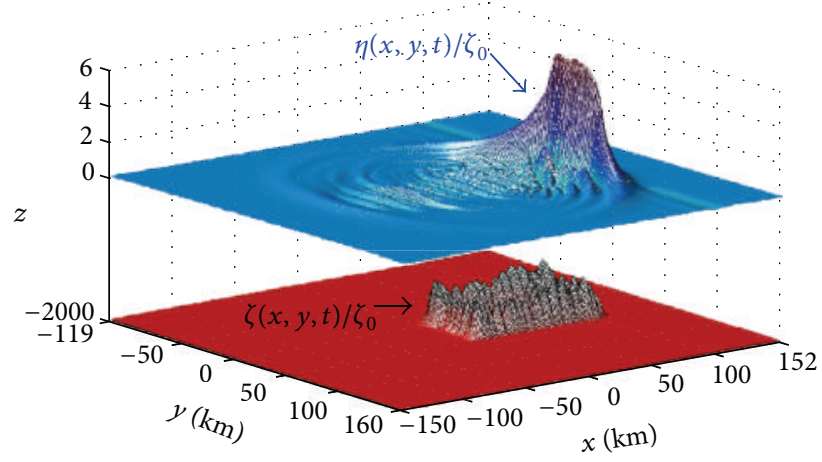

(d) $t=t^{*}$

FIGURE 4: Generation of the normalized tsunami waveforms, $\eta(x, y, t) / \zeta_{0}$, for the same trajectory of the stochastic source model at different rise times with $\sigma_{x}=\sigma_{y}=0.4, L=100 \mathrm{~km}, D=50 \mathrm{~km}, t^{*}=L / v$, and $v=v_{t}$.

Platen [31], can be applied to obtain the mean of the tsunami waveforms as follows:

$$
E[\eta(x, y, t)]=\bar{\eta}(x, y, t)=\eta_{1}(x, y, t) .
$$

Second, to derive the variance of the tsunami waveforms we need to introduce the following operators.

(1) For a function $f\left(t_{1}, t_{2}\right)$, the double Laplace transform $\mathscr{L}_{t_{1} t_{2}}\left\{f\left(t_{1}, t_{2}\right)\right\}$ is defined by

$$
\mathscr{L}_{t_{1} t_{2}}\left\{f\left(t_{1}, t_{2}\right)\right\}=\int_{0}^{\infty} \int_{0}^{\infty} e^{-\left(s_{1} t_{1}+s_{2} t_{2}\right)} f\left(t_{1}, t_{2}\right) d t_{1} d t_{2} .
$$

(2) Let $\mathscr{F}_{x}\{f(x)\}=f^{*}(k)=\int_{-\infty}^{\infty} e^{-i k x} f(x) d x$ represent the Fourier transform of a function $f(x)$. By $\mathscr{F}_{x_{c}}\{f(x)\}$, we mean $\mathscr{F}_{x_{c}}\{f(x)\}=f^{\bullet}(k)=$ $\int_{-\infty}^{\infty} e^{i k x} f(x) d x$

(3) For a function $f(x, y)$, we define $\mathscr{F}_{x y_{c}}\{f(x, y)\}$ by

$$
\begin{aligned}
\mathscr{F}_{x y_{c}}\{f(x, y)\} & =f^{* \bullet}\left(k_{1}, k_{2}\right) \\
& =\int_{-\infty}^{\infty} \int_{-\infty}^{\infty} e^{-i\left(k_{1} x-k_{2} y\right)} f(x, y) d x d y .
\end{aligned}
$$

In addition to the previous operators, the following two lemmas are needed for the derivation of the variance.

\section{Lemma 1. Consider}

$$
\begin{array}{r}
\frac{1}{i k_{1}-i k_{2}+a} f^{*}\left(k_{1}\right) \overline{g^{*}\left(k_{2}\right)} \\
=\mathscr{F}_{x y_{c}}\left\{\int_{0}^{\infty} e^{-a u} f(x-u) \overline{g(y-u)} d u\right\}, \\
k_{2}<k_{1}, \quad a \geq 0,
\end{array}
$$

where $\bar{g}$ denotes the conjugate of the function $g$.

Proof. Consider

$$
\begin{aligned}
& \mathscr{F}_{x y_{c}}\left\{\int_{0}^{\infty} e^{-a u} f(x-u) \overline{g(y-u)} d u\right\} \\
& =\mathscr{F}_{x y_{c}}\left\{\int_{-\infty}^{\infty} e^{-a u} f(x-u) \overline{g(y-u)} H(u) d u\right\} \\
& =\mathscr{F}_{y_{c}}\left\{\mathscr{F}_{x}\left\{\int_{-\infty}^{\infty} f(x-u) e^{-a u} \overline{g(y-u)} H(u) d u\right\}\right\} \\
& =\mathscr{F}_{y_{c}}\left\{f^{*}\left(k_{1}\right) \mathscr{F}_{x}\left\{e^{-a x} \overline{g(y-x)} H(x)\right\}\right\}
\end{aligned}
$$




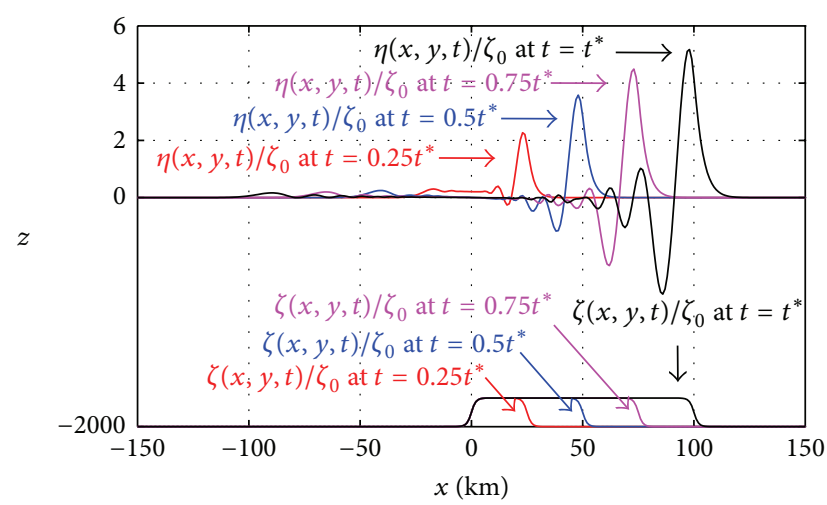

(a) $\sigma_{x}=\sigma_{y}=0$ (deterministic case)

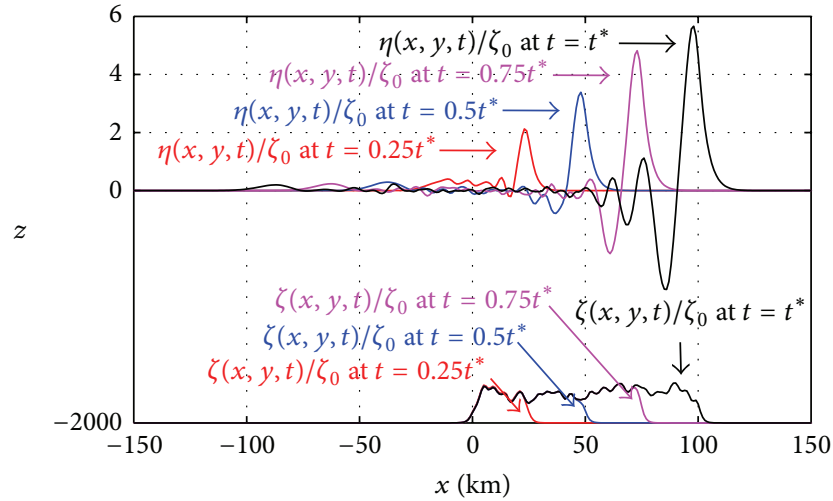

(b) $\sigma_{x}=\sigma_{y}=0.2$

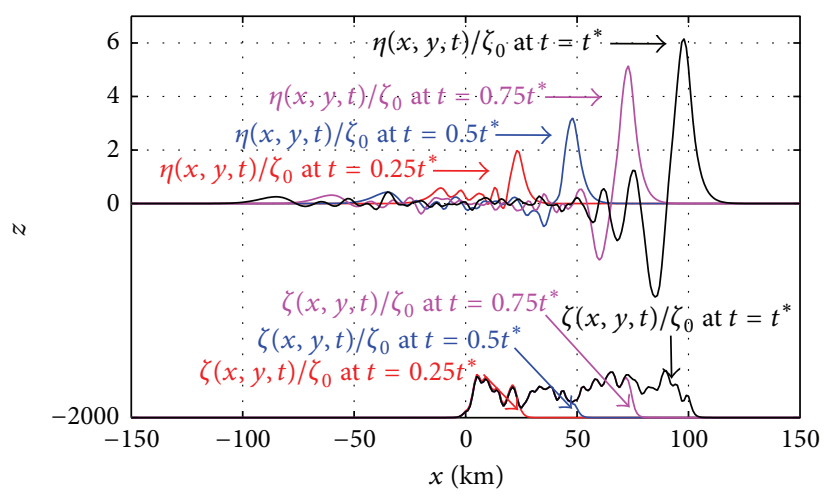

(c) $\sigma_{x}=\sigma_{y}=0.4$

Figure 5: Side view of the generated normalized tsunami waveforms, $\eta(x, y, t) / \zeta_{0}$, for the same trajectory of the stochastic source model at different rise times with noise intensities $\sigma_{x}=\sigma_{y}=0,0.2$ and $0.4, L=100 \mathrm{~km}, D=50 \mathrm{~km}, v=v_{t}$, and $t^{*}=L / v$.

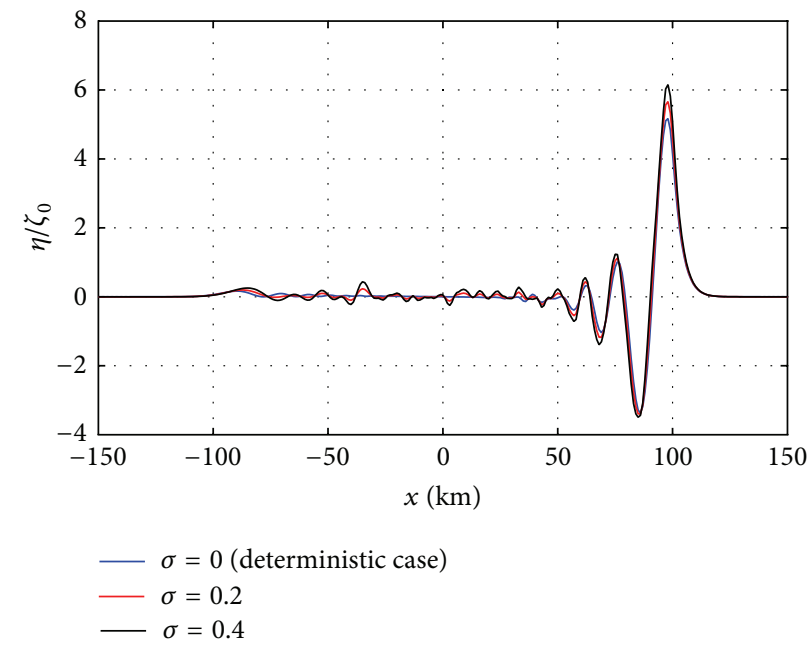

FIGURE 6: Effect of the normalized noise intensities $\sigma_{x}=\sigma_{y}=\sigma$ on the generation of tsunami waveforms for the same trajectory of the stochastic source model with $L=100 \mathrm{~km}$ and $D=50 \mathrm{~km}$ for $t=t^{*}=L / v$. 


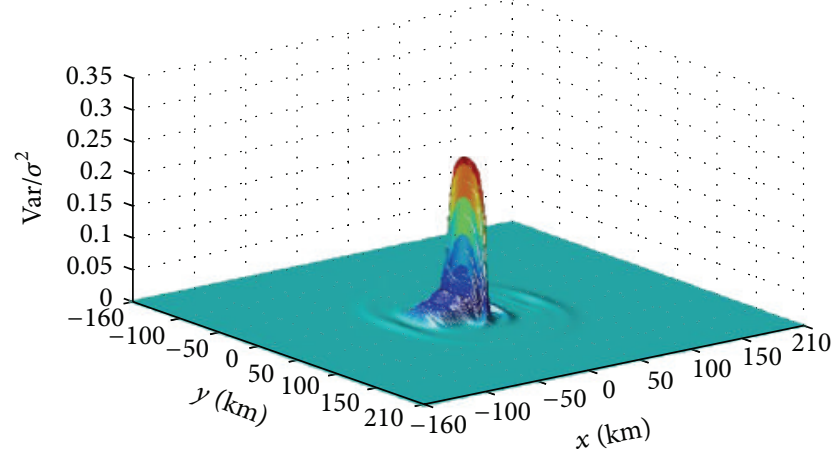

(a) $t=0.25 t^{*}$

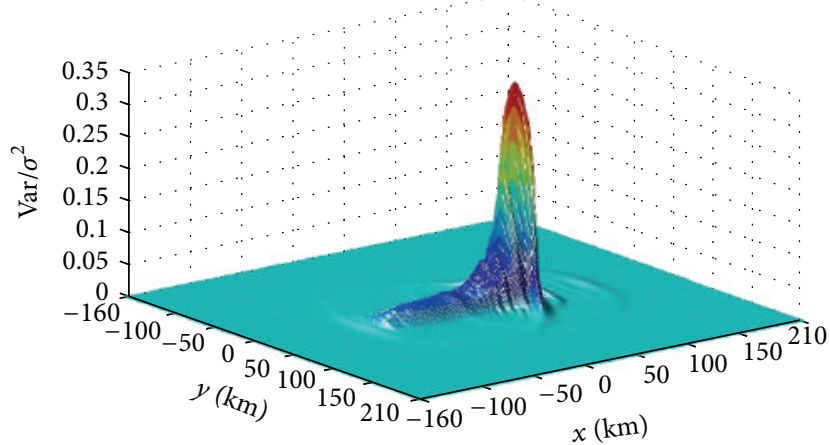

(c) $t=0.75 t^{*}$

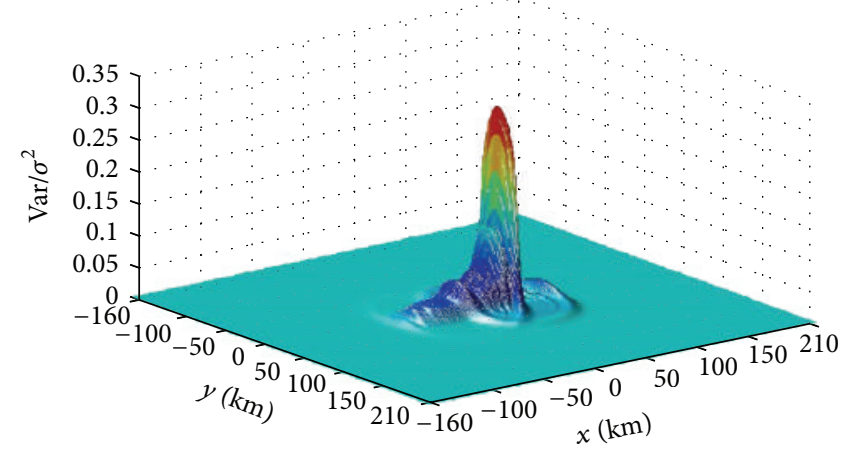

(b) $t=0.5 t^{*}$

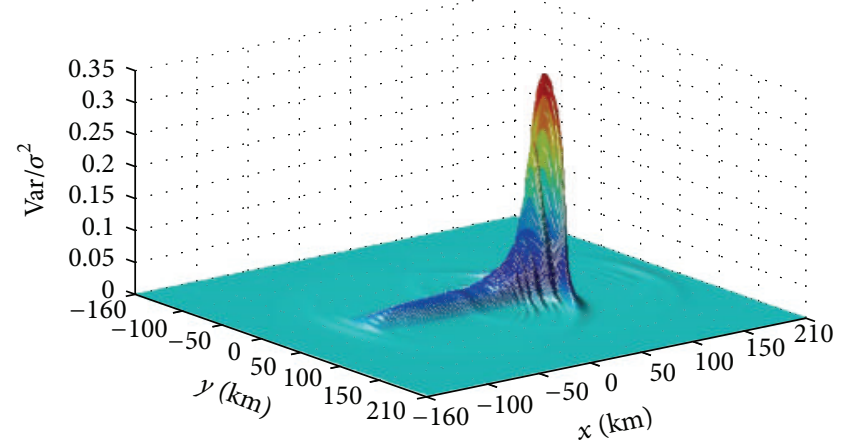

(d) $t=t^{*}$

FiguRE 7: Normalized wave dispersion (variance) of the tsunami generation at different time evolution for $h=2 \mathrm{~km}$ with $L=100 \mathrm{~km}$ and $=50 \mathrm{~km}$.

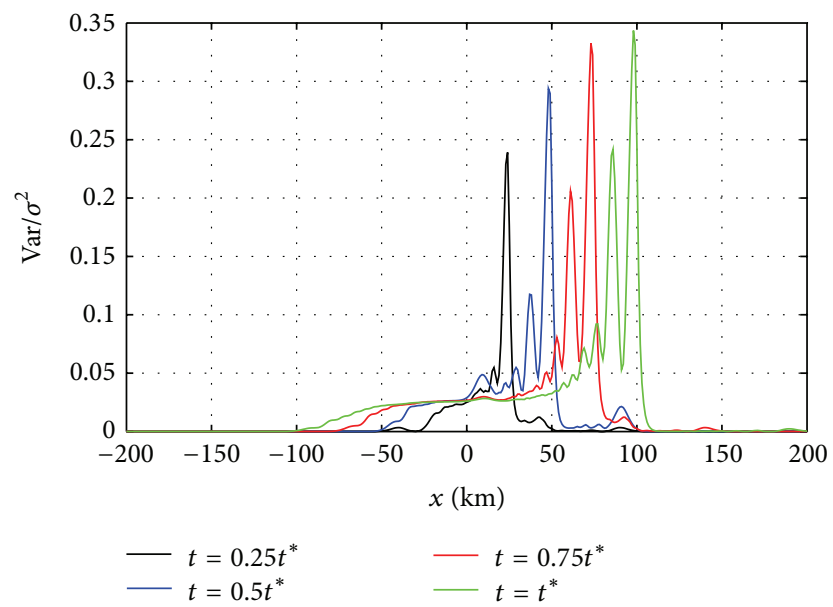

Figure 8: Side view of the normalized wave dispersion of the tsunami generation at different time evolution for $h=2 \mathrm{~km}$ with $L=100 \mathrm{~km}$ and $=50 \mathrm{~km}$.

$$
\begin{array}{lll}
=f^{*}\left(k_{1}\right) \mathscr{F}_{x}\left\{\int_{-\infty}^{\infty} e^{i k_{2} y} e^{-a x} \overline{g(y-x)} H(x) d y\right\} & =f^{*}\left(k_{1}\right) \mathscr{F}_{x}\left\{e^{-a x} H(x) \overline{\left.\int_{-\infty}^{\infty} e^{-i k_{2} y} g(y-x) d y\right\}}\right. \\
=f^{*}\left(k_{1}\right) \mathscr{F}_{x}\left\{e^{-a x} H(x) \int_{-\infty}^{\infty} e^{i k_{2} y} \overline{g(y-x)} d y\right\} & =f^{*}\left(k_{1}\right) \mathscr{F}_{x}\left\{e^{-a x} H(x) \overline{e^{-i k_{2} x} g^{*}\left(k_{2}\right)}\right\}
\end{array}
$$




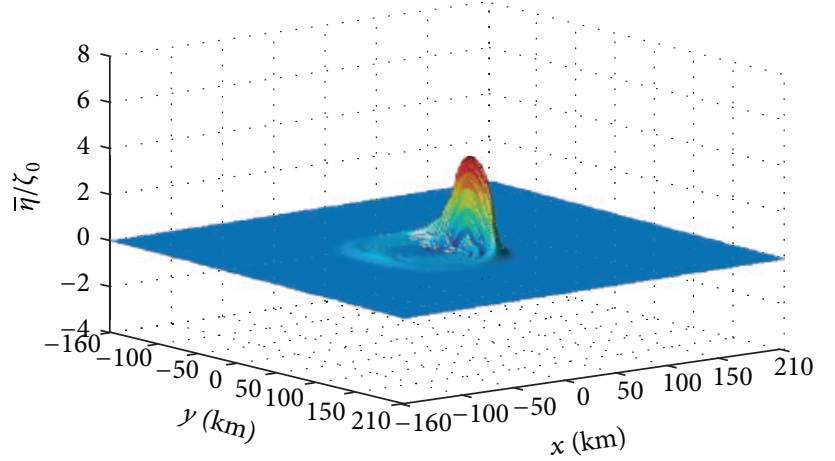

(a) Normalized mean tsunami waveform at $L=50 \mathrm{~km}$

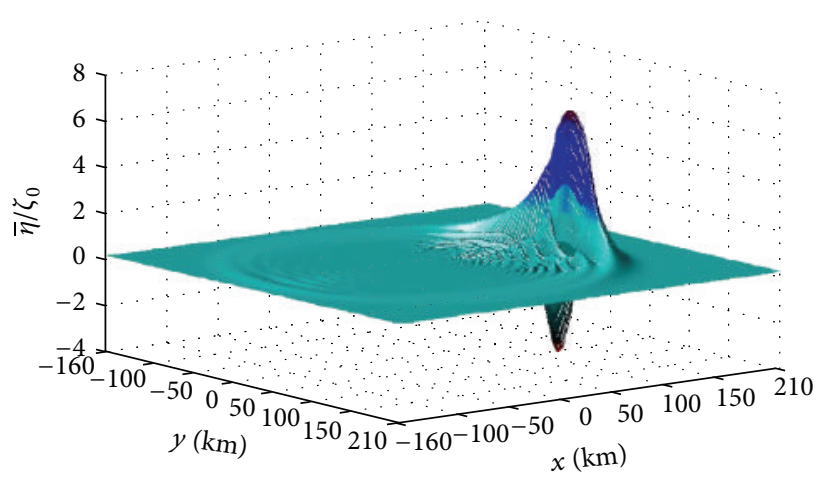

(c) Normalized mean tsunami waveform at $L=150 \mathrm{~km}$

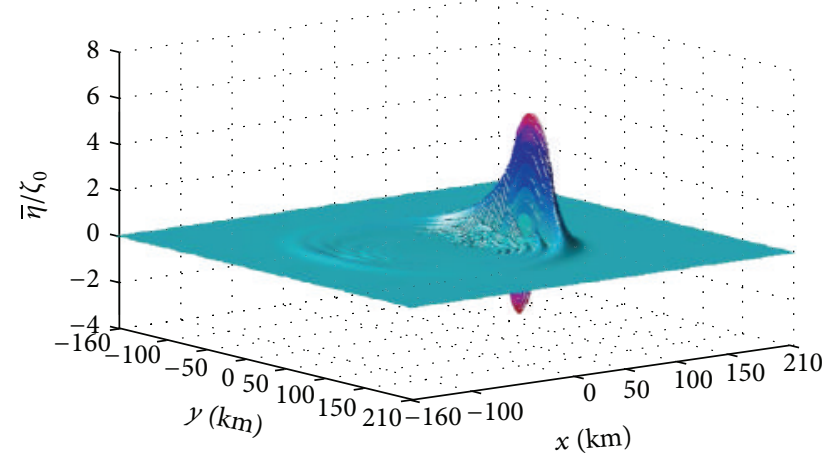

(b) Normalized mean tsunami waveform at $L=100 \mathrm{~km}$

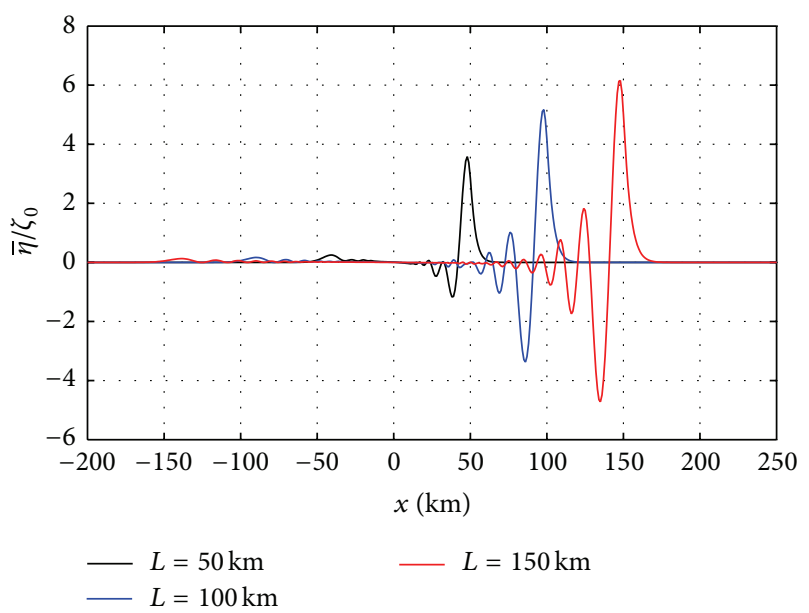

(d) Side view

Figure 9: The normalized mean of the tsunami generated waveforms at different propagated uplift length $L, D=50 \mathrm{~km}, h=2 \mathrm{~km}$, and $t=t^{*}=L / v$.

$$
\begin{aligned}
& =f^{*}\left(k_{1}\right) \overline{g^{*}\left(k_{2}\right)} \int_{-\infty}^{\infty} e^{-i k_{1} x} e^{-a x} H(x) e^{i k_{2} x} d x \\
& =f^{*}\left(k_{1}\right) \overline{g^{*}\left(k_{2}\right)} \int_{0}^{\infty} e^{-\left(i k_{1}-i k_{2}+a\right) x} d x \\
& =\frac{1}{i k_{1}-i k_{2}+a} f^{*}\left(k_{1}\right) \overline{g^{*}\left(k_{2}\right)} .
\end{aligned}
$$

Lemma 2. Consider

$$
\begin{aligned}
& \mathscr{F}_{x x_{c}^{\prime}} \mathscr{F}_{y y_{c}^{\prime}} \mathscr{L}_{t t^{\prime}}\left\{\int_{0}^{\infty} f\left(x-u, y, t-\frac{u}{v}\right) H\left(t-\frac{u}{v}\right)\right. \\
&\left.\times \overline{g\left(x^{\prime}-u, y^{\prime}, t^{\prime}-\frac{u}{v}\right) H\left(t^{\prime}-\frac{u}{v}\right)} d u\right\} \\
&=\frac{1}{i k_{1}-i k_{1}^{\prime}+s / v+s^{\prime} / v} \widehat{f}^{* *}\left(k_{1}, k_{2}, s\right) \overline{\widehat{g}^{* *}\left(k_{1}^{\prime}, k_{2}^{\prime}, s^{\prime}\right) .}
\end{aligned}
$$

\section{Proof. Consider}

$$
\begin{aligned}
& \mathscr{F}_{x x_{c}^{\prime}} \mathscr{F}_{y y_{c}^{\prime}} \mathscr{L}_{t t^{\prime}} \\
& \times\left\{\int_{0}^{\infty} f\left(x-u, y, t-\frac{u}{v}\right) H\left(t-\frac{u}{v}\right)\right. \\
& \left.\times \overline{\left(x^{\prime}-u, y^{\prime}, t^{\prime}-\frac{u}{v}\right) H\left(t^{\prime}-\frac{u}{v}\right)} d u\right\} \\
& =\mathscr{F}_{x x_{c}^{\prime}} \mathscr{F}_{y y_{c}^{\prime}}\left\{\int_{0}^{\infty} \widehat{f}(x-u, y, s) e^{-(s / v) u}\right. \\
& \left.\times \overline{\widehat{g}\left(x^{\prime}-u, y^{\prime}, s^{\prime}\right) e^{-\left(s^{\prime} / v\right) u}} d u\right\} \\
& =\mathscr{F}_{x x_{c}^{\prime}}\left\{\int_{0}^{\infty} e^{-\left(s / v+s^{\prime} / v\right) u} \widehat{f}^{*}\left(x-u, k_{2}, s\right)\right. \\
& \left.\times \overline{\widehat{g}^{*}\left(x^{\prime}-u, k_{2}^{\prime}, s^{\prime}\right)} d u\right\} .
\end{aligned}
$$




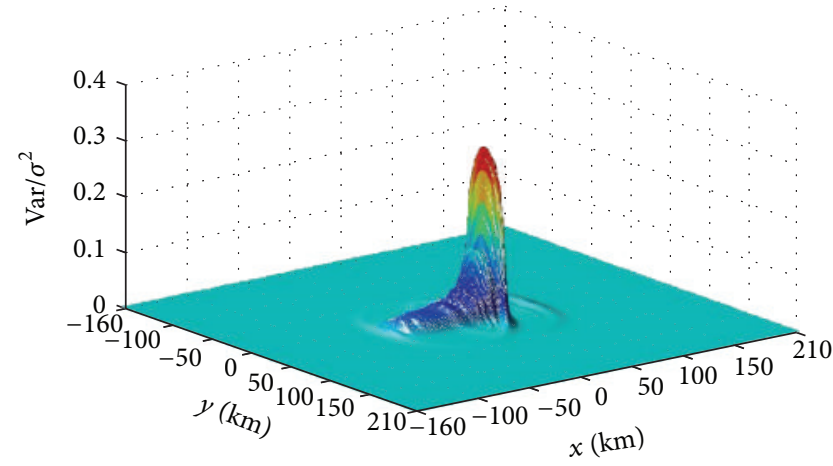

(a) Normalized variance of the tsunami waveforms at $L=50 \mathrm{~km}$

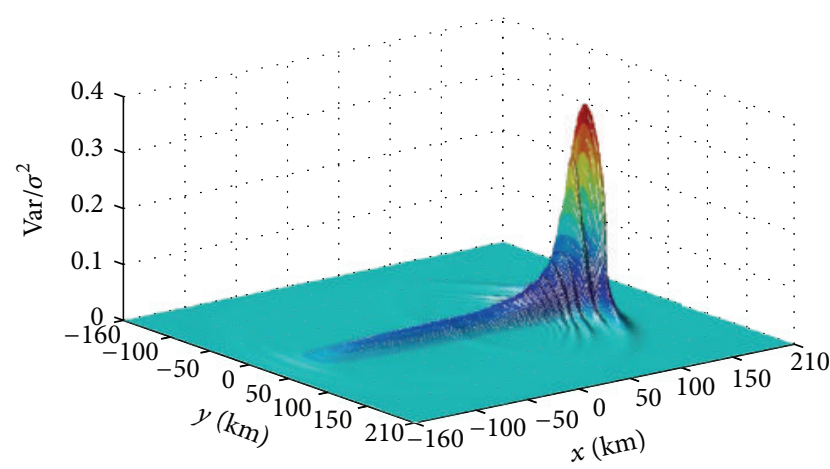

(c) Normalized variance of the tsunami waveforms at $L=150 \mathrm{~km}$

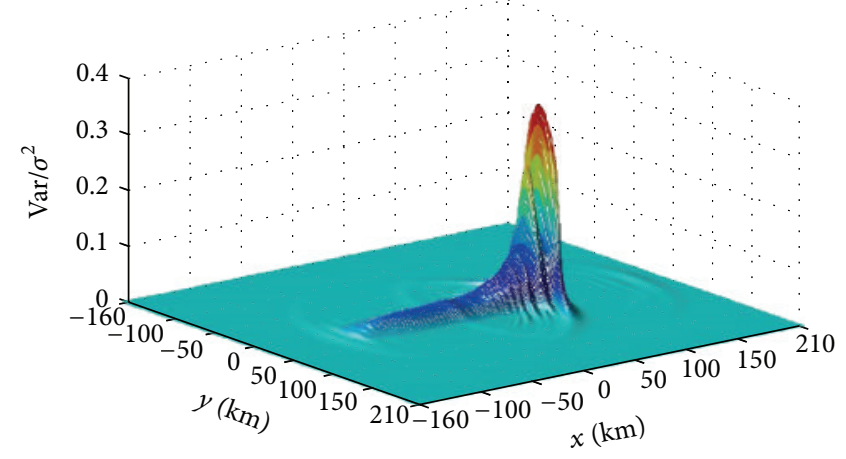

(b) Normalized variance of the tsunami waveforms at $L=100 \mathrm{~km}$

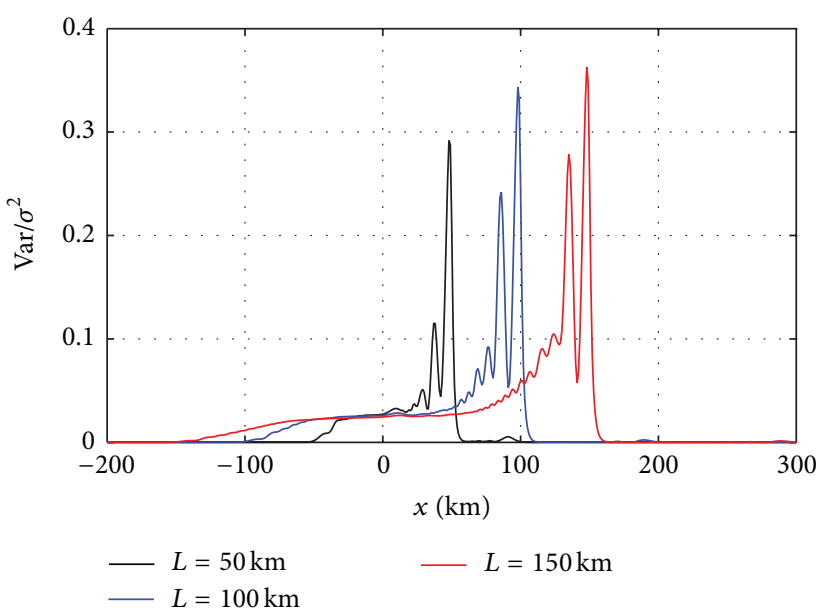

(d) Side view

FIGURE 10: The normalized variance of the tsunami generated waveforms at different propagated uplift length $L, D=50 \mathrm{~km}, h=2 \mathrm{~km}$, and $t=t^{*}=L / v$.

Using Lemma 1, we obtain

$$
\begin{aligned}
\mathscr{F}_{x x_{c}^{\prime}} \mathscr{F}_{y y_{c}^{\prime}} \mathscr{L}_{t t^{\prime}} \\
\times\left\{\int_{0}^{\infty} f\left(x-u, y, t-\frac{u}{v}\right) H\left(t-\frac{u}{v}\right)\right. \\
\left.\quad \times \overline{g\left(x^{\prime}-u, y^{\prime}, t^{\prime}-\frac{u}{v}\right) H\left(t^{\prime}-\frac{u}{v}\right)} d u\right\} \\
=\frac{1}{i k_{1}-i k_{1}^{\prime}+s / v+s^{\prime} / v} \\
\quad \times \widehat{f}^{* *}\left(k_{1}, k_{2}, s\right) \overline{\widehat{g}^{* *}\left(k_{1}^{\prime}, k_{2}^{\prime}, s^{\prime}\right) .}
\end{aligned}
$$

Let $R_{\Omega \overline{\Omega^{\prime}}}\left(x, y, t, x^{\prime}, y^{\prime}, t^{\prime}\right)=E\left[\Omega(x, y, t) \overline{\Omega^{\prime}\left(x^{\prime}, y^{\prime}, t^{\prime}\right)}\right]$ denote the autocorrelation function of the process $\Omega(x, y, t)$. It can be shown that

$$
\begin{aligned}
\mathscr{F}_{x x_{c}^{\prime}} \mathscr{F}_{y y_{c}^{\prime}} \mathscr{L}_{t t^{\prime}}\left\{R_{\Omega \overline{\Omega^{\prime}}}\left(x, y, t, x^{\prime}, y^{\prime}, t^{\prime}\right)\right\} \\
=E\left[\widehat{\Omega}^{* *}\left(k_{1}, k_{2}, s\right) \overline{\left.{\widehat{\Omega^{\prime}}}^{* *}\left(k_{1}^{\prime}, k_{2}^{\prime}, s^{\prime}\right)\right] .}\right.
\end{aligned}
$$

Assuming that $\widehat{\Omega}^{* *}\left(k_{1}, k_{2}, s\right)$ is the difference between the transformed free surface elevation (34) and its mean (36),

$$
\begin{aligned}
\widehat{\Omega}^{* *} & \left(k_{1}, k_{2}, s\right) \\
= & \widehat{\alpha}_{x}^{* *}\left(k_{1}, k_{2}, s\right) \int_{0}^{L} e^{-\left(i k_{1}+s / v\right) x} d W(x) \\
& +\widehat{\alpha}_{y}^{* *}\left(k_{1}, k_{2}, s\right) \int_{0}^{D} e^{-i k_{2} y} d W(y),
\end{aligned}
$$

then

$$
\begin{aligned}
E\left[\widehat{\Omega}^{* *}\right. & \left.\left(k_{1}, k_{2}, s\right) \overline{\widehat{\Omega}^{\prime * *}\left(k_{1}^{\prime}, k_{2}^{\prime}, s^{\prime}\right)}\right] \\
=E & {\left[\left(\widehat{\alpha}_{x}^{* *}\left(k_{1}, k_{2}, s\right) \int_{0}^{L} e^{-\left(i k_{1}+s / v\right) x} d W(x)\right)\right.} \\
& \times\left(\widehat{\alpha}_{y}^{* *}\left(k_{1}, k_{2}, s\right) \int_{0}^{D} e^{-i k_{2} y} d W(y)\right) \\
& \times\left(\overline{\widehat{\alpha}_{y}^{\prime *}}\left(k_{1}^{\prime}, k_{2}^{\prime}, s^{\prime}\right) \int_{0}^{D} e^{-i k_{2} y} d W(y)\right) \\
& \left.\times\left(\overline{\widehat{\alpha}_{y}^{* *}}\left(k_{1}^{\prime}, k_{2}^{\prime}, s^{\prime}\right) \int_{0}^{D} e^{i k_{2} y} d W(y)\right)\right]
\end{aligned}
$$




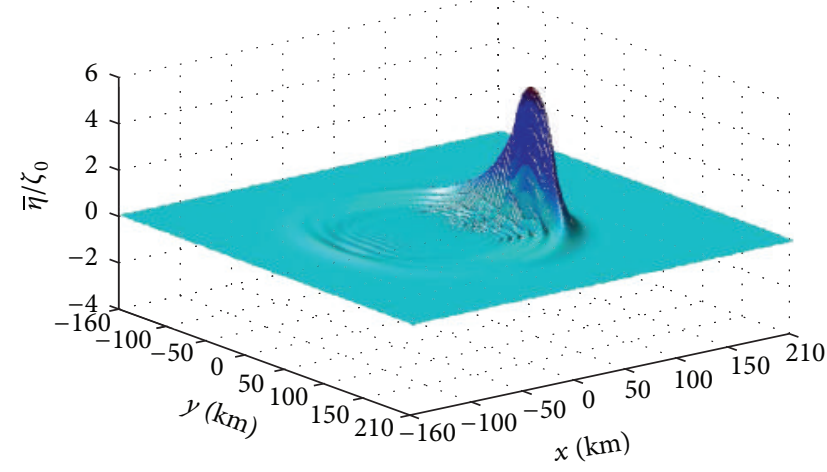

(a) Normalized mean tsunami waveform at $h=2 \mathrm{~km}$

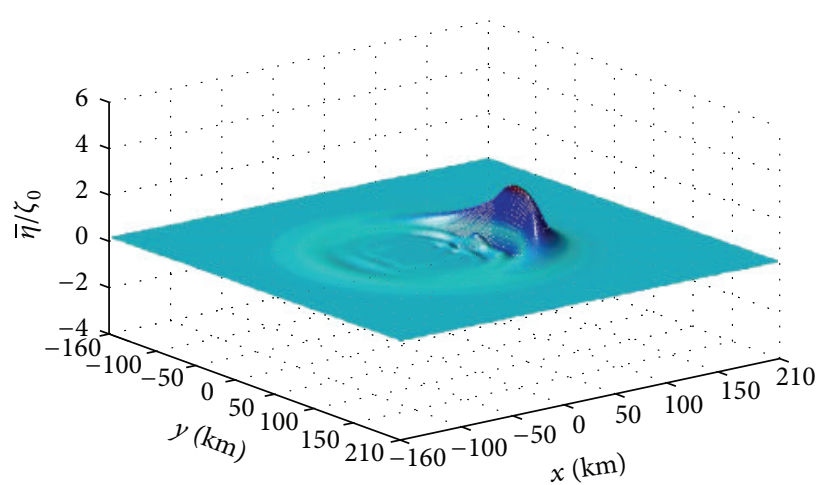

(c) Normalized mean tsunami waveform at $h=6 \mathrm{~km}$

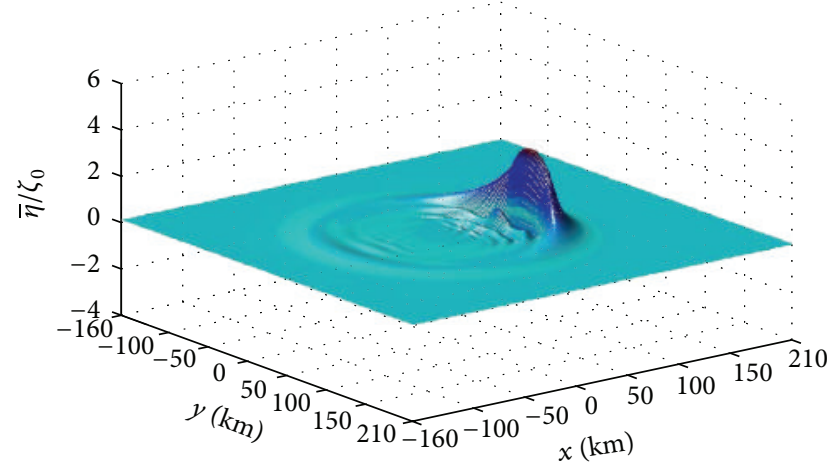

(b) Normalized mean tsunami waveform at $h=4 \mathrm{~km}$

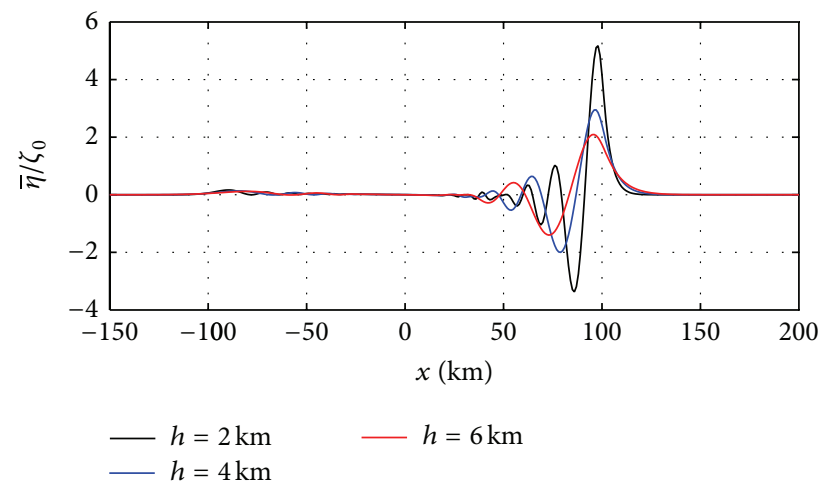

(d) Side view

FIGURE 11: Effect of the water depth $h$ on the normalized mean of the generated tsunami waveforms at $L=100 \mathrm{~km}$ and $D=50 \mathrm{~km}$ at time $t=t^{*}=L / v$.

$$
\begin{aligned}
& =\left(\widehat{\alpha}_{x}^{* *}\left(k_{1}, k_{2}, s\right){\widehat{\widehat{\alpha}^{\prime}}}_{x}^{* *}\left(k_{1}^{\prime}, k_{2}^{\prime}, s^{\prime}\right)\right) \\
& \times E\left[\left(\int_{0}^{L} e^{-\left(i k_{1}+s / v\right) x} d W(x)\right)\right. \\
& \left.\times\left(\int_{0}^{L} e^{\left(i k_{1}^{\prime}-s^{\prime} / v\right) x} d W(x)\right)\right] \\
& +\left(\widehat{\alpha}_{y}^{* *}\left(k_{1}, k_{2}, s\right){\widehat{\widehat{\alpha}^{\prime}}}_{y}^{* *}\left(k_{1}^{\prime}, k_{2}^{\prime}, s^{\prime}\right)\right) \\
& \times E\left[\left(\int_{0}^{D} e^{-i k_{2} y} d W(y)\right)\left(\int_{0}^{D} e^{i k_{2} y} d W(y)\right)\right] .
\end{aligned}
$$

Recalling the fact, see [30, Theorem 4.5, page 99], that if $X(t)$ and $Y(t)$ are regular adapted processes, such that

$$
E\left[\int_{0}^{T} X^{2}(t) d t\right]<\infty, \quad E\left[\int_{0}^{T} Y^{2}(t) d t\right]<\infty
$$

then we have

$$
\begin{aligned}
E & {\left[\left(\int_{0}^{T} X(t) d W(t)\right)\left(\int_{0}^{T} Y(t) d W(t)\right)\right] } \\
& =\int_{0}^{T} E[X(t) Y(t)] d t,
\end{aligned}
$$

and hence (47) becomes

$$
\begin{aligned}
E\left[\widehat{\Omega}^{* *}\left(k_{1}, k_{2}, s\right) \overline{{\widehat{\Omega^{\prime}}}^{* *}\left(k_{1}^{\prime}, k_{2}^{\prime}, s^{\prime}\right)}\right] \\
=\widehat{\alpha}_{x}^{* *}\left(k_{1}, k_{2}, s\right) \overline{{\widehat{\alpha^{\prime}}}_{x}^{* *}\left(k_{1}^{\prime}, k_{2}^{\prime}, s^{\prime}\right)} \\
\quad \times \int_{0}^{L} e^{-\left(i k_{1}-i k_{1}^{\prime}+s / v+s^{\prime} / v\right) x} d x \\
\quad+\widehat{\alpha}_{y}^{* *}\left(k_{1}, k_{2}, s\right){\overline{\widehat{\alpha}^{\prime}}}_{y}^{* *}\left(k_{1}^{\prime}, k_{2}^{\prime}, s^{\prime}\right) \\
\quad \times \int_{0}^{D} e^{-\left(i k_{2}-i k_{2}^{\prime}\right) y} d y
\end{aligned}
$$




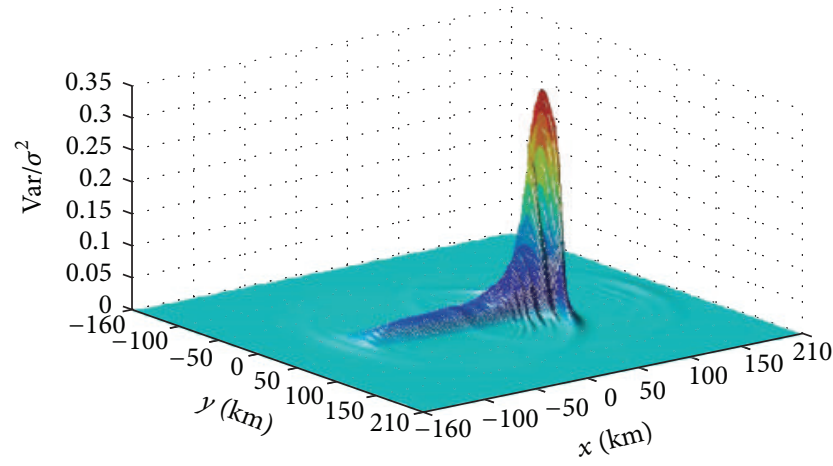

(a) Normalized variance of the tsunami waveforms at $h=2 \mathrm{~km}$

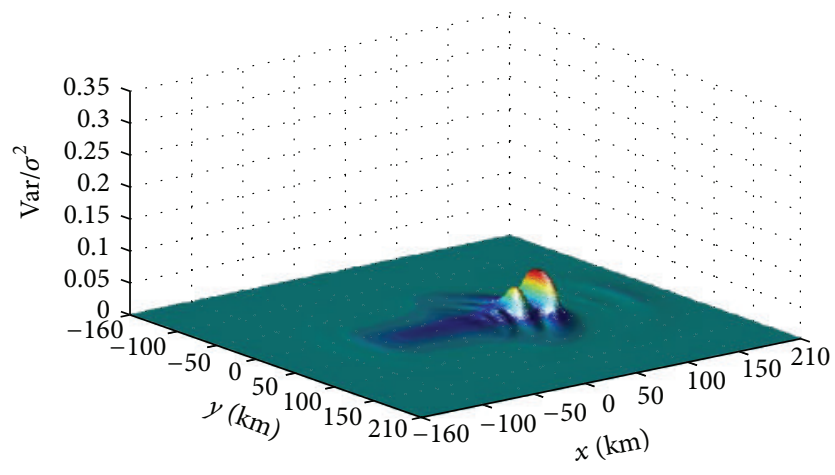

(c) Normalized variance of the tsunami waveforms at $h=6 \mathrm{~km}$

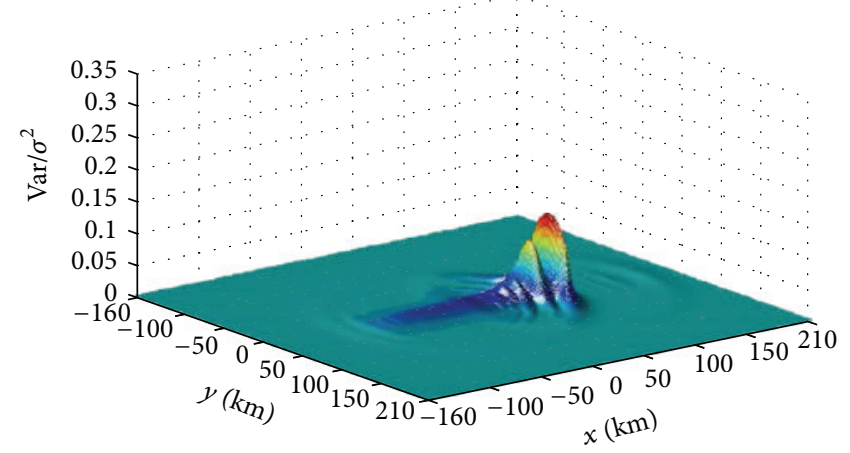

(b) Normalized variance of the tsunami waveforms at $h=4 \mathrm{~km}$

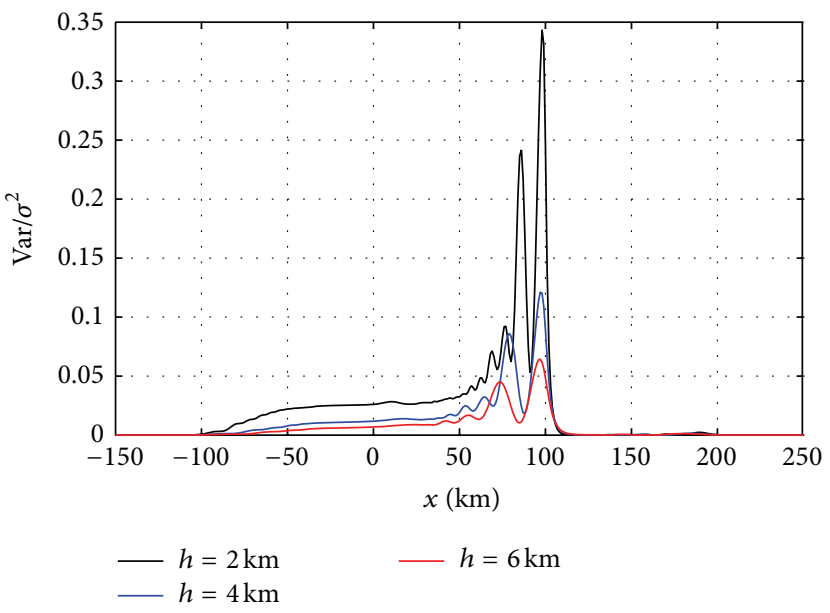

(d) Side view

FIGURE 12: Effect of the water depth $h$ on the normalized variance of the generated tsunami waveforms at $L=100 \mathrm{~km}$ and $D=50 \mathrm{~km}$ at time $t=t^{*}=L / v$.

$$
\begin{aligned}
= & \widehat{\alpha}_{x}^{* *}\left(k_{1}, k_{2}, s\right) \overline{{\widehat{\alpha^{\prime}}}_{x}^{* *}\left(k_{1}^{\prime}, k_{2}^{\prime}, s^{\prime}\right)} \\
& \times \frac{1-e^{-\left(i k_{1}-i k_{1}^{\prime}+s / v+s^{\prime} / v\right) L}}{\left(i k_{1}-i k_{1}^{\prime}+s / v+s^{\prime} / v\right)} \\
& +\widehat{\alpha}_{y}^{* *}\left(k_{1}, k_{2}, s\right){\widehat{\widehat{\alpha}_{y}^{\prime * *}}\left(k_{1}^{\prime}, k_{2}^{\prime}, s^{\prime}\right)} \\
& \times \frac{1-e^{-\left(i k_{2}-i k_{2}^{\prime}\right) D}}{\left(i k_{2}-i k_{2}^{\prime}\right)} \\
= & \frac{1}{\left(i k_{1}-i k_{1}^{\prime}+s / v+s^{\prime} / v\right)}
\end{aligned}
$$

$$
\begin{gathered}
\times\left[\widehat{\alpha}_{x}^{* *}\left(k_{1}, k_{2}, s\right) \widehat{\alpha_{x}^{\prime * *}\left(k_{1}^{\prime}, k_{2}^{\prime}, s^{\prime}\right)}\right. \\
-\left(\widehat{\alpha}_{x}^{* *}\left(k_{1}, k_{2}, s\right) e^{-\left(i k_{1}+s / v\right) L}\right) \\
\times \frac{\left({\widehat{\alpha^{\prime}}}_{x}^{* *}\left(k_{1}^{\prime}, k_{2}^{\prime}, s^{\prime}\right) e^{-\left(i k_{1}^{\prime}+s^{\prime} / v\right) L}\right)}{+\frac{1}{\left(i k_{2}-i k_{2}^{\prime}\right)}\left[\widehat{\alpha}_{y}^{* *}\left(k_{1}, k_{2}, s\right)\right.}
\end{gathered}
$$




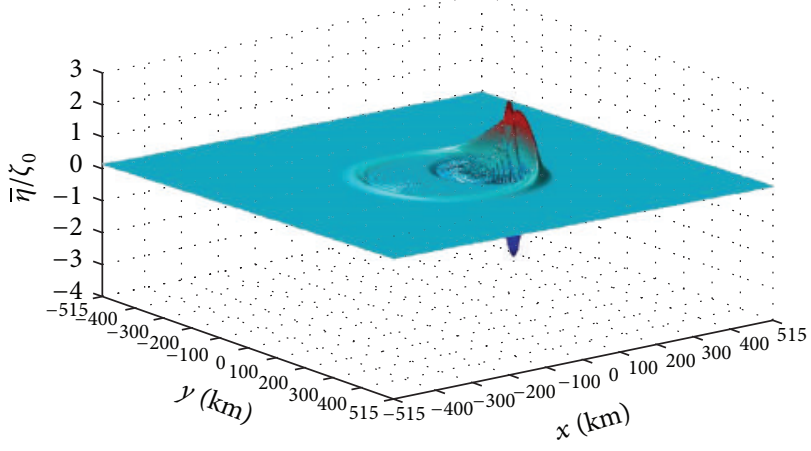

(a) $t=2 t^{*}$

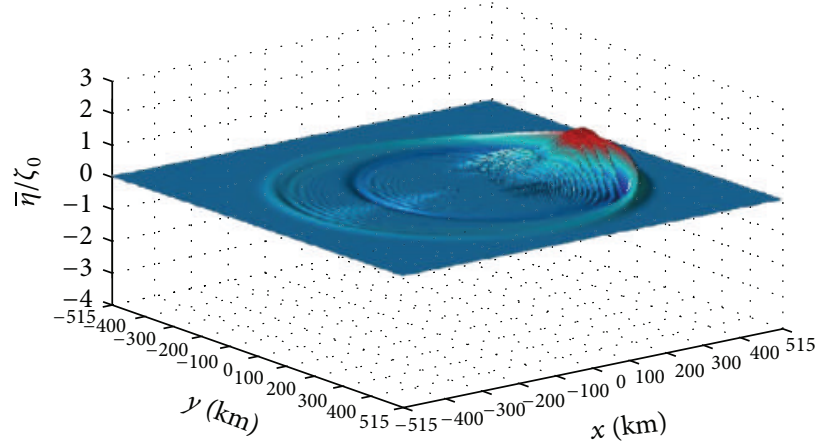

(c) $t=4 t^{*}$

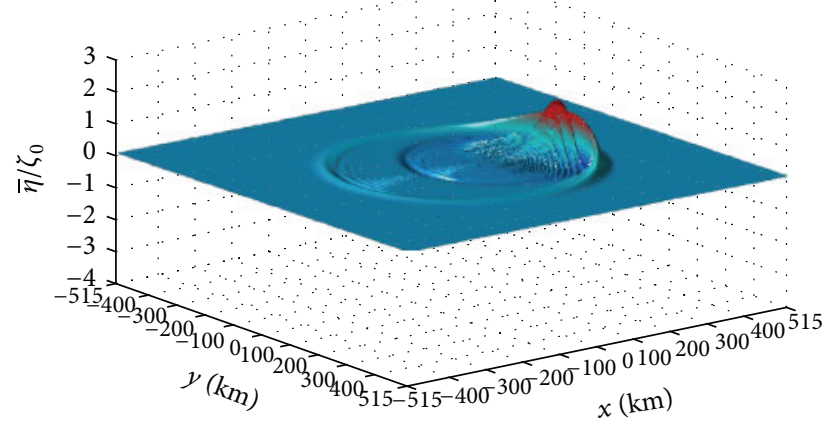

(b) $t=3 t^{*}$

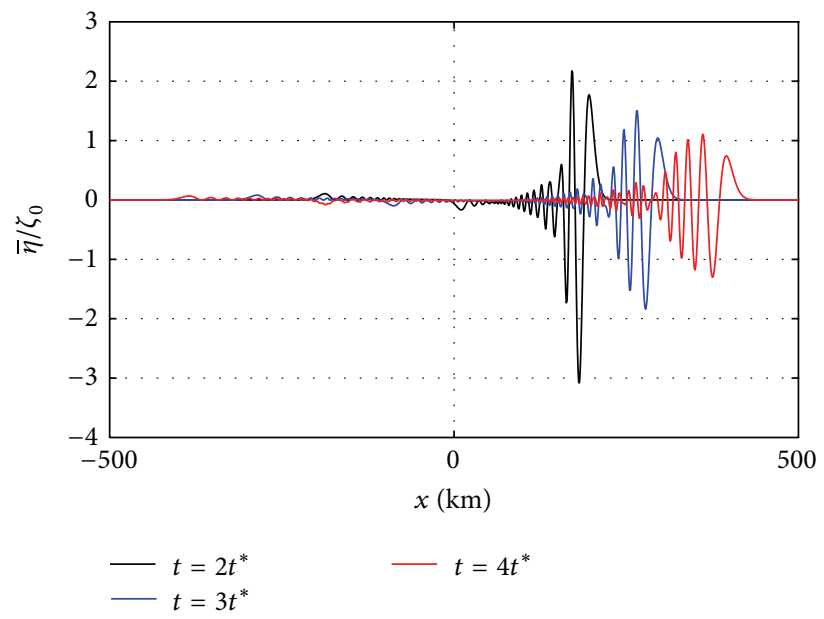

(d) Side view at $t=2 t^{*}, 3 t^{*}, 4 t^{*}$

FIGURE 13: The normalized mean of the propagated tsunami waveforms at different propagation times for propagated uplift length $L=100 \mathrm{~km}$, width $D=50 \mathrm{~km}, h=2 \mathrm{~km}$ at $t=2 t^{*}, 3 t^{*}, 4 t^{*}$, where $t^{*}=L / v$ and $v=v_{t}$.

$$
\begin{aligned}
& \times{\widehat{\widehat{\alpha}_{y}^{\prime}}}_{y}^{* *}\left(k_{1}^{\prime}, k_{2}^{\prime}, s^{\prime}\right) \\
& -\left(\widehat{\alpha}_{y}^{* *}\left(k_{1}, k_{2}, s\right) e^{-i k_{2} D}\right)
\end{aligned}
$$$$
\left.\times \overline{\left({\widehat{\alpha^{\prime}}}_{y}^{* *}\left(k_{1}^{\prime}, k_{2}^{\prime}, s^{\prime}\right) e^{-i k_{2}^{\prime} D}\right)}\right] .
$$

Applying Lemma 2 to (50), we obtain

$$
\begin{aligned}
& E\left[\widehat{\Omega}^{* *}\left(k_{1}, k_{2}, s\right) \overline{{\widehat{\Omega^{\prime}}}^{* *}\left(k_{1}^{\prime}, k_{2}^{\prime}, s^{\prime}\right)}\right] \\
& =\mathscr{F}_{x x_{c}^{\prime}} \mathscr{F}_{y y_{c}^{\prime}} \mathscr{L}_{t t^{\prime}} \\
& \quad \times\left\{\int_{0}^{\infty} \alpha_{x}\left(x-u, y, t-\frac{u}{v}\right) H\left(t-\frac{u}{v}\right) \overline{\alpha_{x}\left(x^{\prime}-u, y^{\prime}, t^{\prime}-\frac{u}{v}\right) H\left(t^{\prime}-\frac{u}{v}\right)} d u\right. \\
& \quad-\int_{0}^{\infty} \alpha_{x}\left(x-L-u, y, t-\frac{L+u}{v}\right) H\left(t-\frac{L+u}{v}\right) H\left(t-\frac{u}{v}\right) \\
& \quad+\int_{0}^{\infty} \alpha_{y}(x, y-u, t) \overline{\alpha_{y}\left(x^{\prime}, y^{\prime}-u, t^{\prime}\right)} d u \\
& \left.\quad-\int_{0}^{\infty} \alpha_{y}(x, y-D-u, t) \overline{\alpha_{y}\left(x^{\prime}, y^{\prime}-D-u, t^{\prime}\right)} d u\right\} .
\end{aligned}
$$




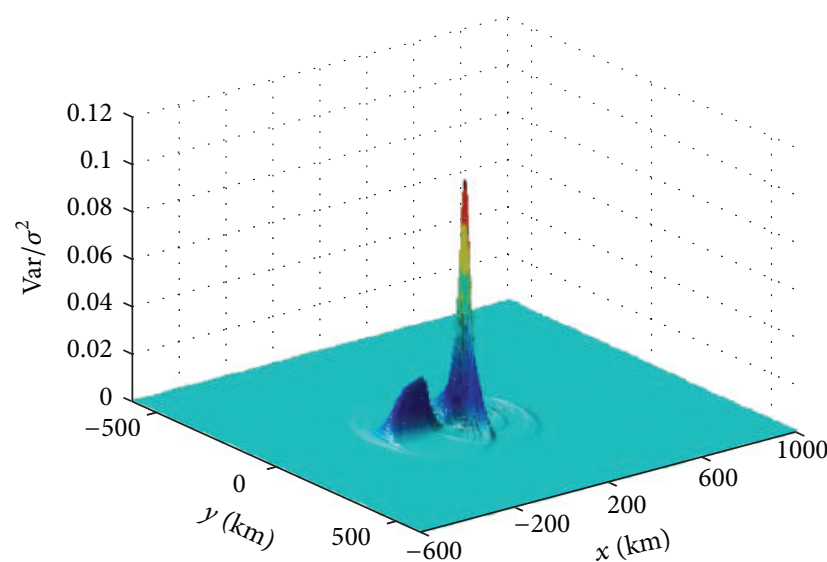

(a) $t=2 t^{*}$

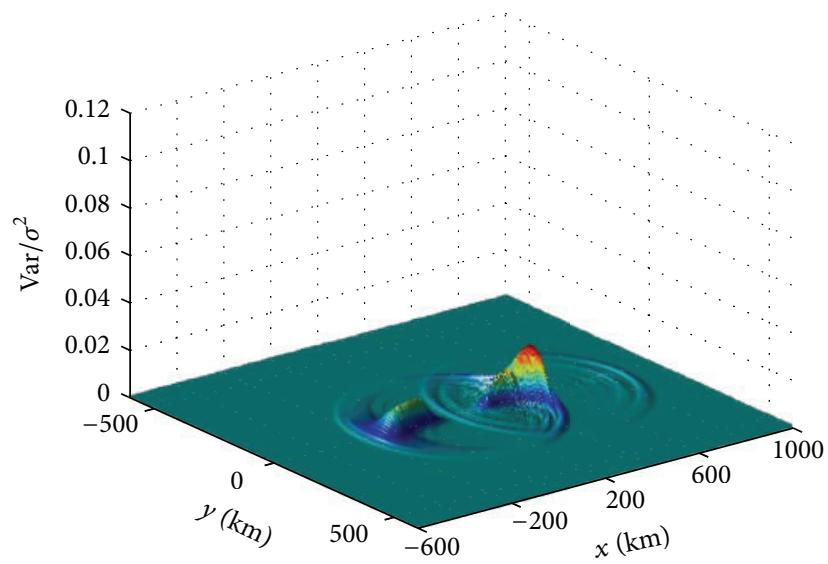

(c) $t=4 t^{*}$

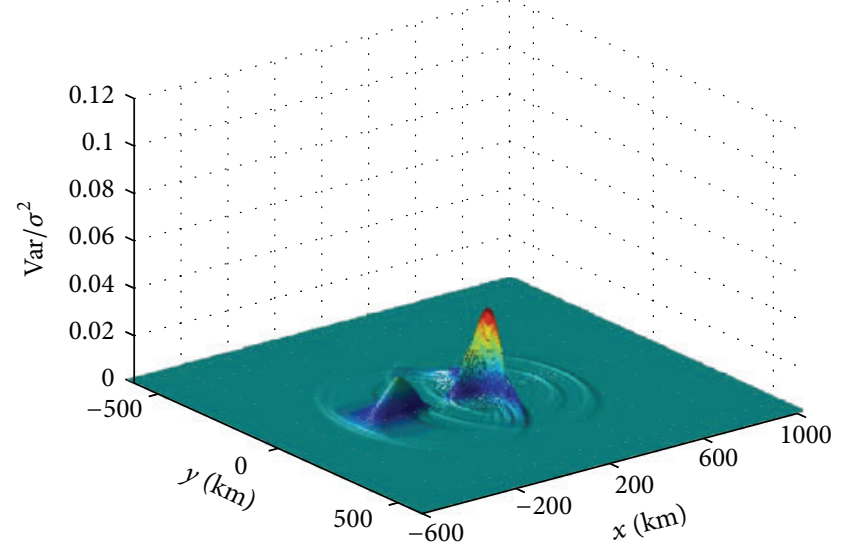

(b) $t=3 t^{*}$

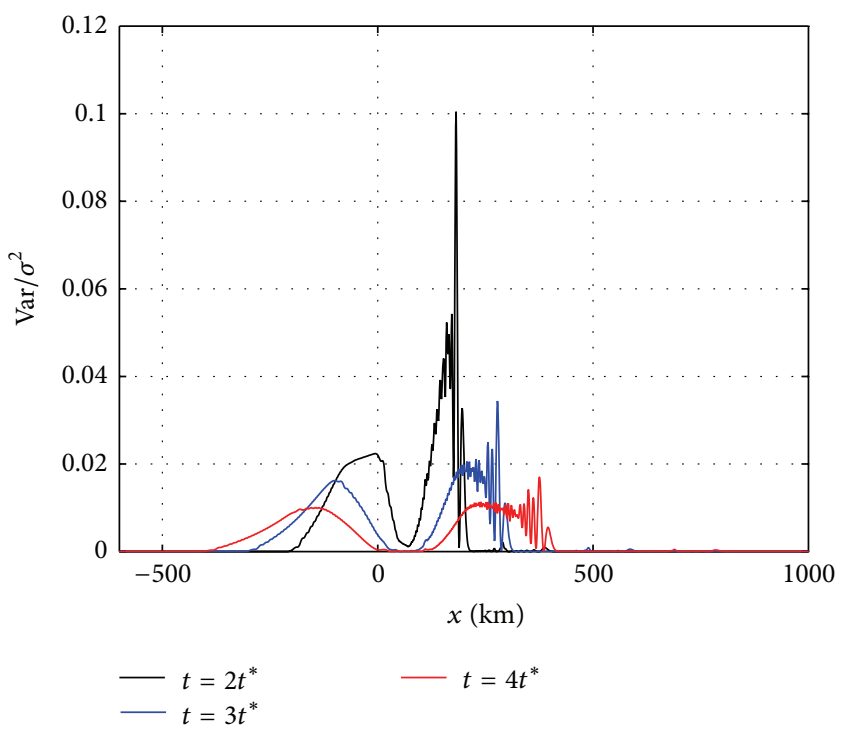

(d) Side view at $t=2 t^{*}, 3 t^{*}, 4 t^{*}$

FIGURE 14: The normalized variance of the propagated tsunami waveforms at different propagation times for propagated uplift length $L=$ $100 \mathrm{~km}$, width $D=50 \mathrm{~km}, h=2 \mathrm{~km}$ at $t=2 t^{*}, 3 t^{*}, 4 t^{*}$, where $t^{*}=L / v$ and $v=v_{t}$.

From (45) and (51), we can deduce that

$$
\begin{aligned}
& R_{\Omega \overline{\Omega^{\prime}}}\left(x, y, t, x^{\prime}, y^{\prime}, t^{\prime}\right) \\
& =\int_{0}^{\infty} \alpha_{x}\left(x-u, y, t-\frac{u}{v}\right) H\left(t-\frac{u}{v}\right) \overline{\alpha_{x}\left(x^{\prime}-u, y^{\prime}, t^{\prime}-\frac{u}{v}\right) H\left(t^{\prime}-\frac{u}{v}\right)} d u \\
& \quad-\int_{0}^{\infty} \frac{\alpha_{x}\left(x-L-u, y, t-\frac{L+u}{v}\right) H\left(t-\frac{L+u}{v}\right) H\left(t-\frac{u}{v}\right)}{\quad} \overline{\times \alpha_{x}\left(x^{\prime}-L-u, y^{\prime}, t^{\prime}-\frac{L+u}{v}\right) H\left(t^{\prime}-\frac{L+u}{v}\right) H\left(t^{\prime}-\frac{u}{v}\right)} d u
\end{aligned}
$$




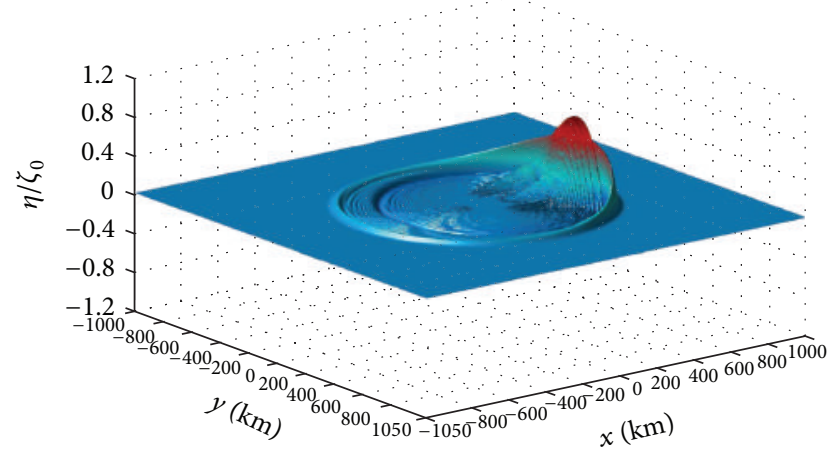

(a) $t=4 t^{*}$

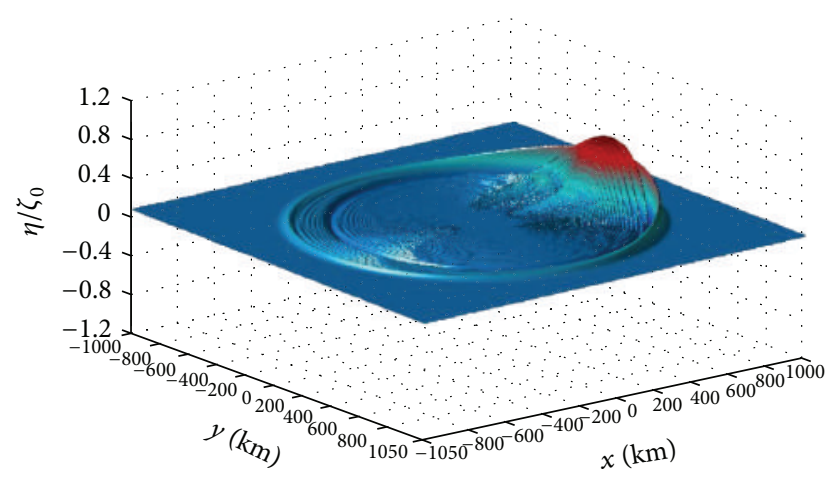

(c) $t=8 t^{*}$

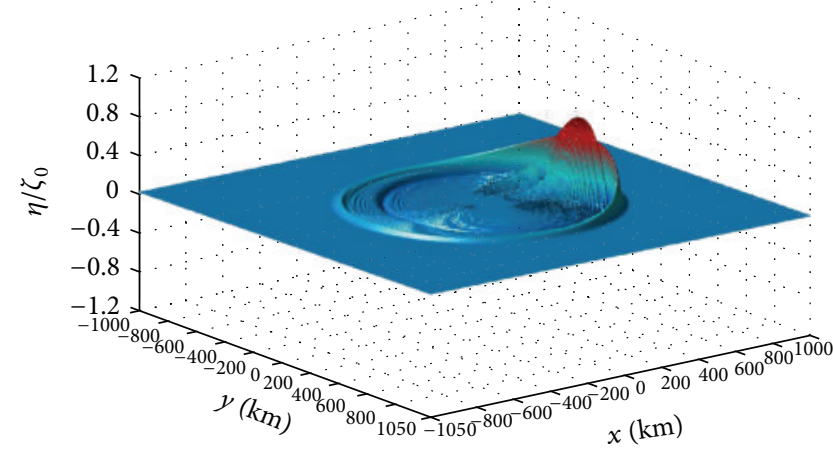

(b) $t=6 t^{*}$

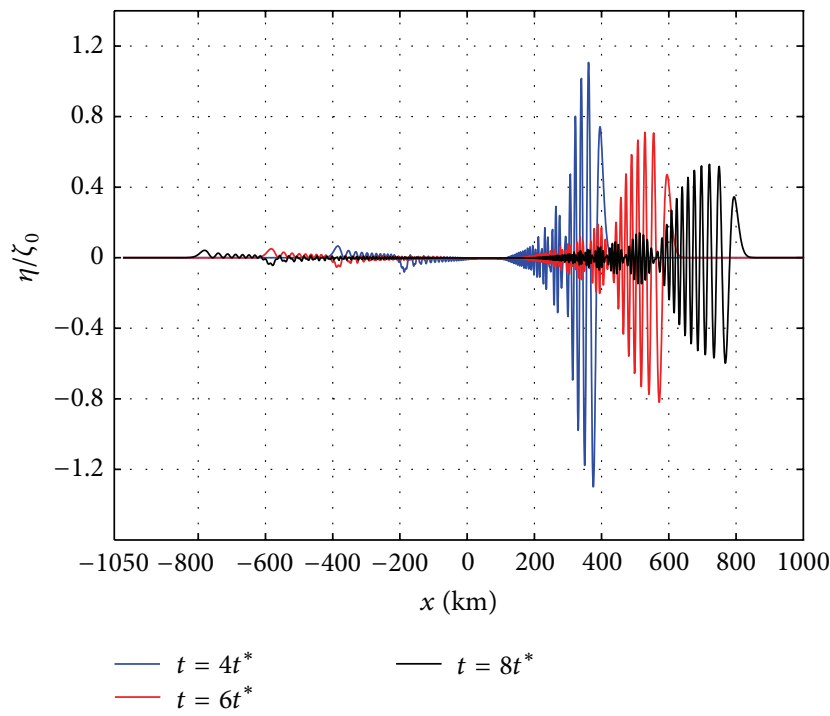

(d) Side view at $t=4 t^{*}, 6 t^{*}, 8 t^{*}$

Figure 15: Propagation of the normalized tsunami waveforms, $\eta(x, y, t) / \zeta_{0}$, for different rise times at $\sigma_{x}=\sigma_{y}=0, L=100 \mathrm{~km}, D=50 \mathrm{~km}$, $v=v_{t}$, and $t^{*}=L / v$.

$$
\begin{aligned}
& +\int_{0}^{\infty} \alpha_{y}(x, y-u, t) \overline{\alpha_{y}\left(x^{\prime}, y^{\prime}-u, t^{\prime}\right)} d u \\
& -\int_{0}^{\infty} \alpha_{y}(x, y-D-u, t) \overline{\alpha_{y}\left(x^{\prime}, y^{\prime}-D-u, t^{\prime}\right)} d u .
\end{aligned}
$$

Finally, the variance can be obtained by taking $x^{\prime}=x, y^{\prime}=y$, and $t^{\prime}=t$ in the autocorrelation function (52) as follows:

$$
\begin{aligned}
\operatorname{Var} & {[\Omega(x, y, t)] } \\
= & R_{\Omega \bar{\Omega}}(x, y, t) \\
= & \int_{0}^{\infty}\left|\alpha_{x}\left(x-u, y, t-\frac{u}{v}\right) H\left(t-\frac{u}{v}\right)\right|^{2} d u \\
& -\int_{0}^{\infty} \mid \alpha_{x}\left(x-L-u, y, t-\frac{L+u}{v}\right)
\end{aligned}
$$

$$
\begin{gathered}
\quad \times\left. H\left(t-\frac{L+u}{v}\right) H\left(t-\frac{u}{v}\right)\right|^{2} d u \\
+\int_{0}^{\infty}\left(\left|\alpha_{y}(x, y-u, t)\right|^{2}\right. \\
\left.\quad-\left|\alpha_{y}(x, y-D-u, t)\right|^{2}\right) d u \\
=\int_{0}^{t v}\left|\alpha_{x}\left(x-u, y, t-\frac{u}{v}\right)\right|^{2} d u \\
+\int_{0}^{\infty}\left(\left|\alpha_{y}(x, y-u, t)\right|^{2}\right.
\end{gathered}
$$




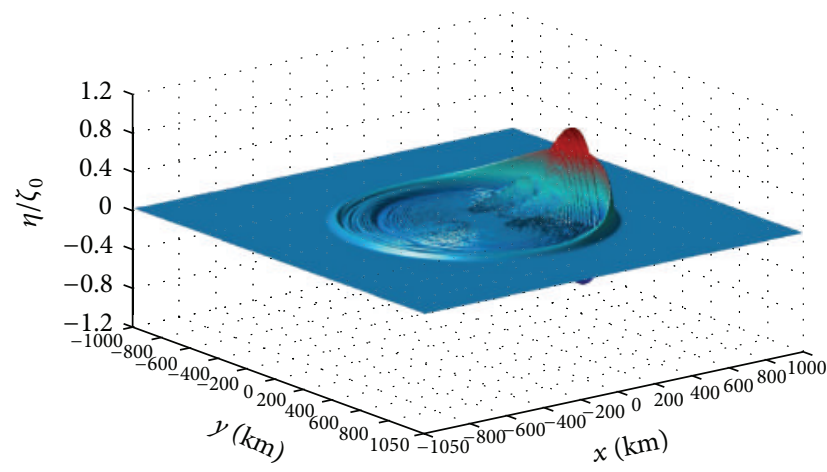

(a) $t=4 t^{*}$

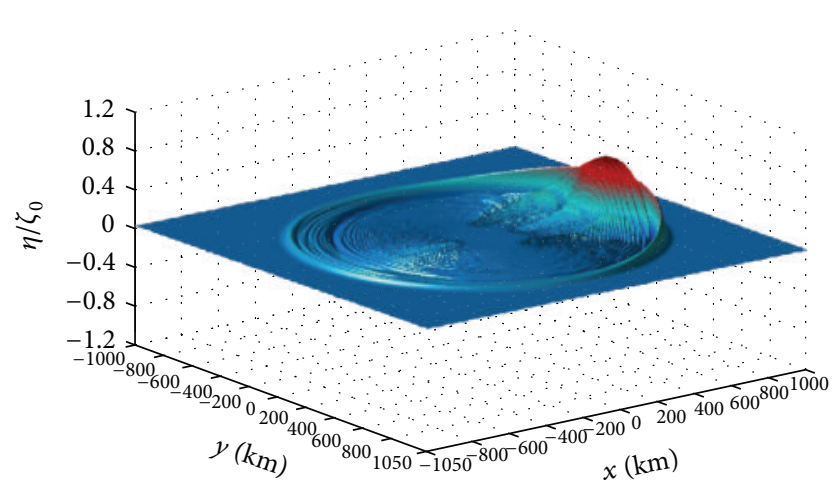

(c) $t=8 t^{*}$

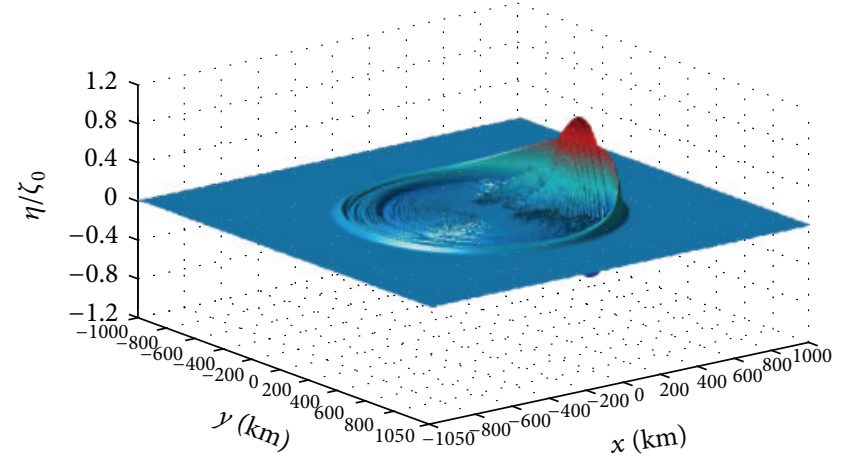

(b) $t=6 t^{*}$

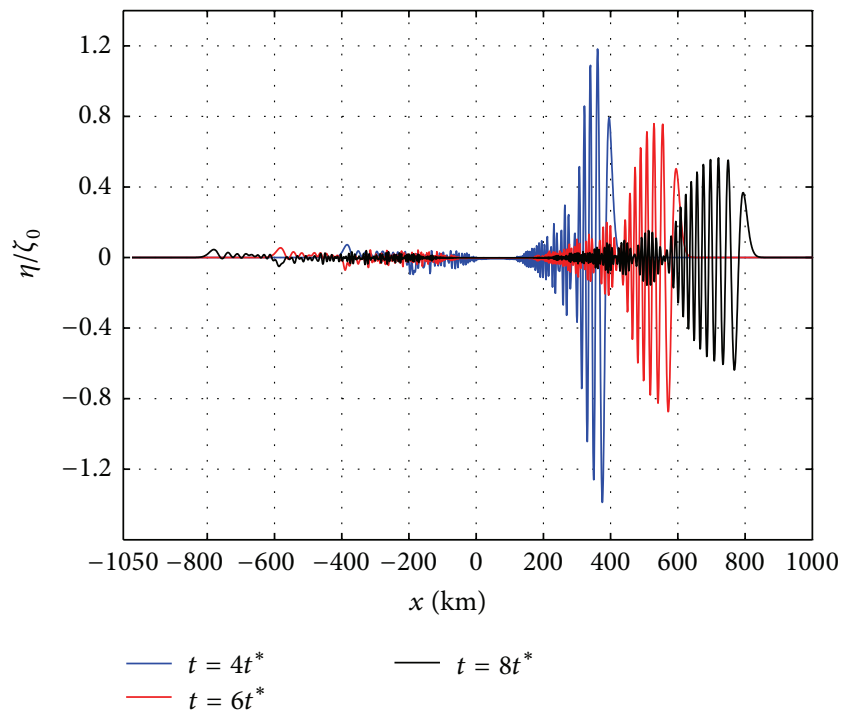

(d) Side view at $t=4 t^{*}, 6 t^{*}, 8 t^{*}$

Figure 16: Propagation of the normalized tsunami waveforms, $\eta(x, y, t) / \zeta_{0}$, for the same trajectory of the stochastic source model at different rise times with $\sigma_{x}=\sigma_{y}=0.2, L=100 \mathrm{~km}, D=50 \mathrm{~km}, v=v_{t}$, and $t^{*}=L / v$.

$$
\begin{gathered}
\left.-\left|\alpha_{y}(x, y-D-u, t)\right|^{2}\right) d u \\
-\left\{\begin{array}{l}
\int_{0}^{t v-L}\left|\alpha_{x}\left(x-L-u, y, t-\frac{L+u}{v}\right)\right|^{2} d u, \\
\text { for } t>t^{*} \\
\int_{0}^{t v}\left|\alpha_{x}\left(x-L-u, y, t-\frac{L+u}{v}\right)\right|^{2} d u, \\
\text { for } t \leq t^{*} .
\end{array}\right.
\end{gathered}
$$

\section{Results and Discussion}

In this section, we present numerical results to illustrate the nature of the tsunami build-up and propagation during and after the uplift process of a stochastic bottom topography. This model was used to evaluate tsunami waveforms up to distances of several source dimensions, for various combinations of the model parameters (depth to the sea bed, $h$, fault length and width, $L$ and $D$, and time since rupture initiation, $t$ ). The numerical results demonstrate the waveform amplification resulting from source spreading in the $x$-direction and wave focusing in the near-field and the tsunami spreading in the far-field under the effect of different factors. When the source process is completed and for rapid lateral spreading, the displacement of the free surface above the source resembles the displacement of the ocean floor; see $[10,18]$. For velocities of spreading smaller than $v_{t}$, the tsunami amplitudes in the direction of the source propagation become small with high frequencies. As the velocity of the spreading approaches $v_{t}$, the tsunami waveform has progressively larger amplitude, with high frequency content, in the direction of the slip spreading; see $[10,18]$. These large amplitudes are caused by wave focusing (i.e., during slow earthquakes). Examples of such slow earthquakes are the June 6, 1960, Chile earthquakes which ruptured as a series of earthquakes for about an hour, see [36], and the February 21, 1978, Banda Sea earthquake; see [37].

Tsunami generally occurs due to vertical movement of the seafloor that vertically displaces the water column. Large vertical displacement of the sea bottom ground causes a 


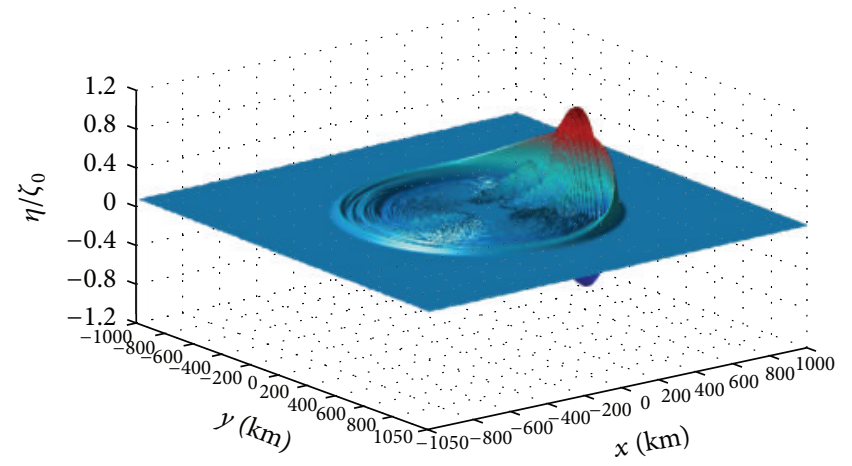

(a) $t=4 t^{*}$

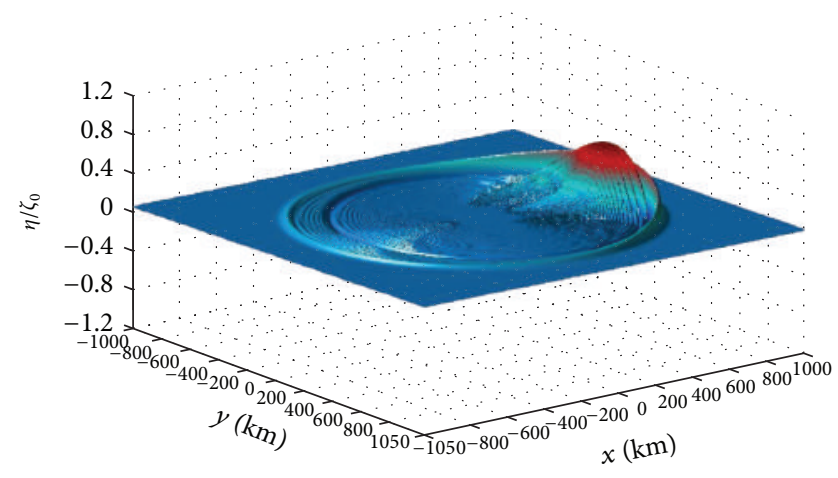

(c) $t=8 t^{*}$

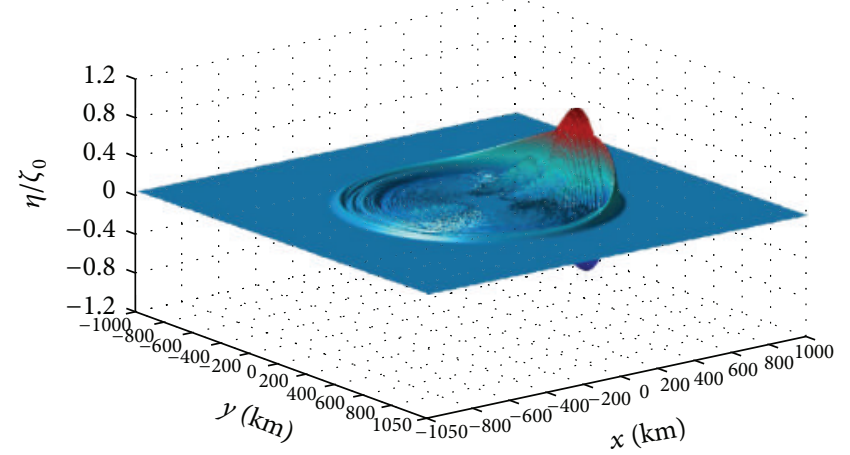

(b) $t=6 t^{*}$

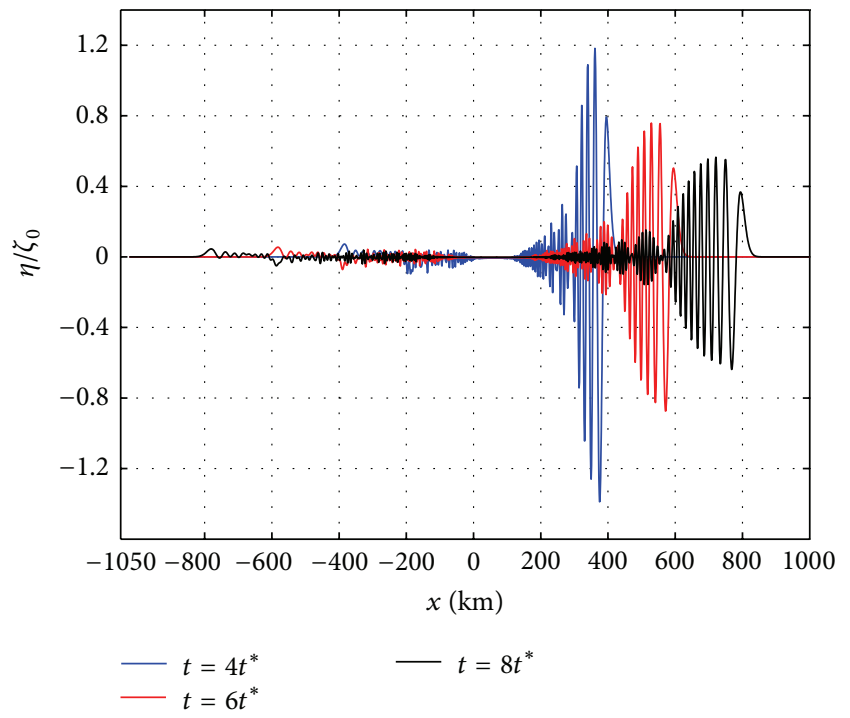

(d) Side view at $t=4 t^{*}, 6 t^{*}, 8 t^{*}$

Figure 17: Propagation of the normalized tsunami waveforms, $\eta(x, y, t) / \zeta_{0}$, for the same trajectory of the stochastic source model at different rise times with $\sigma_{x}=\sigma_{y}=0.4, L=100 \mathrm{~km}, D=50 \mathrm{~km}, v=v_{t}$, and $t^{*}=L / v$.

corresponding large motion at the sea surface. The generation of tsunami by vertical displacements of the ocean floor depends on the characteristic size (length $L$ and width $D$ ) of the displaced area and on the time $t$ it takes to spread the motion over the entire source region. Therefore, researchers presented kinematic source models in the form of Heaviside functions to describe the generation of tsunamis; see [9$12,38,39]$.

It is difficult to estimate, at present, how often the amplification may occur during actual faulting, sliding, or slumping, because of the lack of detailed knowledge about the ground deformations in the source area of past tsunamis. Therefore, we constructed mathematically a reasonable random tsunami source model represented by a sliding Heaviside step function under the influence of two independent Gaussian white noise processes in the $x$ - and $y$-directions. Due to the stochastic nature of the tsunami waveforms, we mainly examine the mean and variance of the tsunami generation and propagation under the effect of different factors.
3.1. Time Evolution during Tsunami Generation. The effects of variations of the uplift of the considered stochastic source model in the $x$ - and $y$-directions are studied as a function of time $t$ on the generation of tsunamis. These effects are studied through the investigation of the generation of tsunamis by unilateral displacements of the ocean floor and under the effect of the characteristic size length $L$ of the displaced area, water depth $h$, and normalized noise intensities $\sigma_{x}$ and $\sigma_{y}$, where $\sigma_{x}=\sigma_{x_{0}} / \zeta_{0}$ and $\sigma_{y}=\sigma_{y_{0}} / \zeta_{0}$. The velocity of the sea floor spreads, $v$, is chosen to be equal to the long wave tsunami velocity $v_{t}=\sqrt{g h}$, (i.e., maximum amplification). The tsunami waveforms generated for the stochastic source model with normalized noise intensities $\sigma_{x}=\sigma_{y}=0$ (deterministic case), 0.2 , and 0.4 are illustrated in Figures 2,3 , and 4 , respectively. These waveforms are generated at constant water depth $h=2 \mathrm{~km}$, propagated length $L=$ $100 \mathrm{~km}$, and source width $D=50 \mathrm{~km}$ at rise time $t=$ $0.25 t^{*}, 0.5 t^{*}, 0.75 t^{*}$, and $t^{*}$, where $t^{*}=L / v$ and $v=v_{t}$. It can be seen from these figures that the amplitude of the 


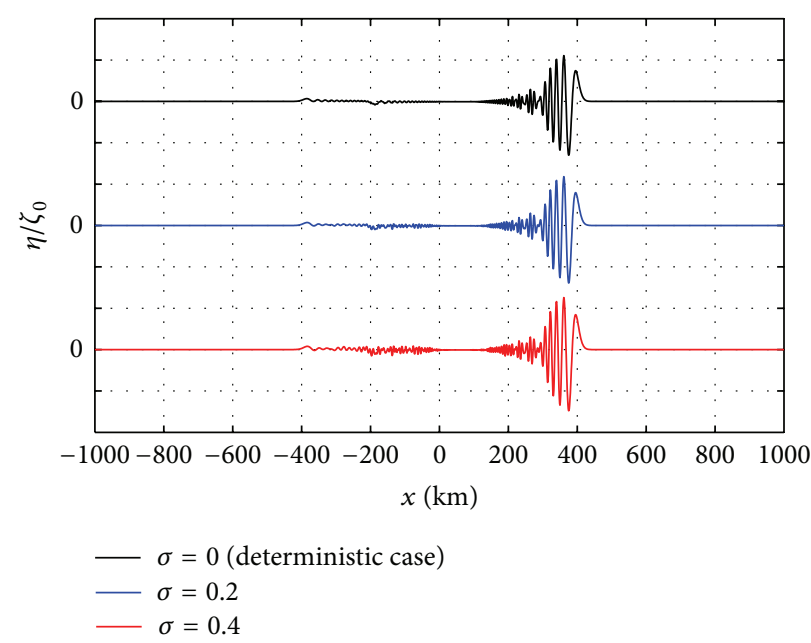

(a) $t=4 t^{*}$

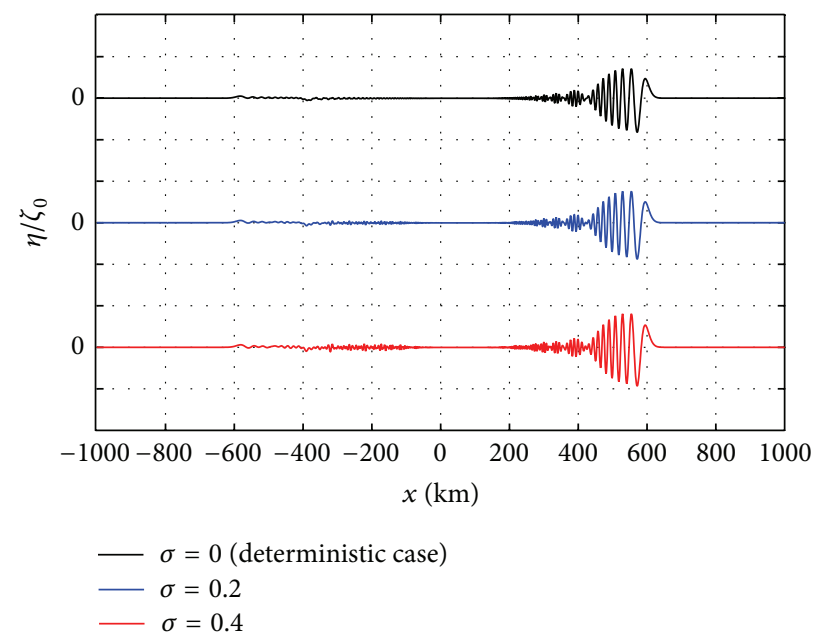

(b) $t=6 t^{*}$

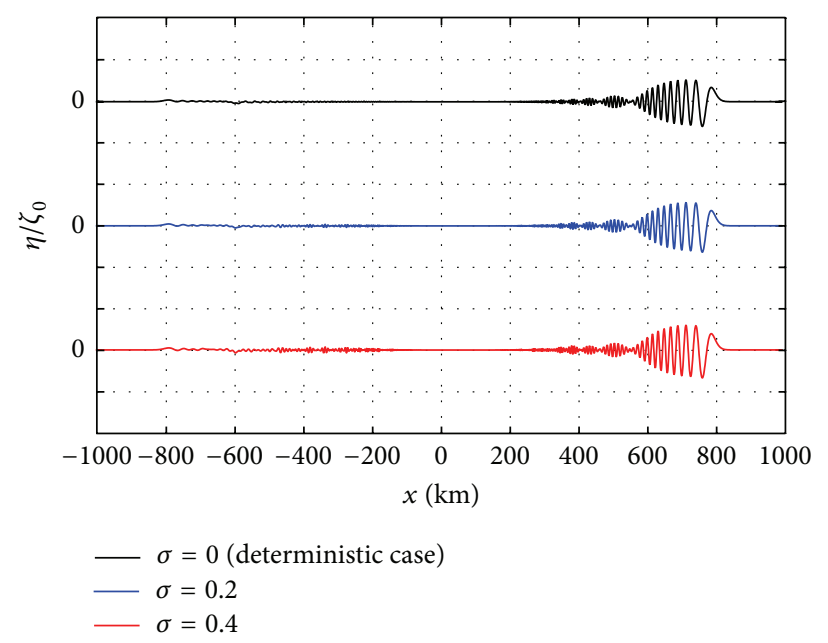

(c) $t=8 t^{*}$

FIGURE 18: Effect of the normalized noise intensities $\sigma_{x}$ and $\sigma_{y}$ on the propagation of tsunami waveforms for the same trajectory of the stochastic source model with $L=100 \mathrm{~km}$ and $D=50 \mathrm{~km}$ for different propagation times $t$, where $t^{*} 100 / v$.

wave builds up progressively as $t$ increases and the focusing and the amplification of tsunami amplitudes occur above the spreading edge of the source model as seen in Figure 5. This amplification occurs above the source progressively, as the source evolves, by adding uplifted fluid to the fluid displaced previously by uplifts of preceding source segments. As the spreading length in the fault increases, the amplitude of the tsunami wave increases. Moreover, it can be observed from Figure 5 that, at $t=0.5 t^{*}$, the deterministic waveform is in complete agreement with the aspect of the tsunami generated by a slowly spreading uplift of the ocean bottom presented by Todorovska and Trifunac [10] who considered a very simple kinematic source model on the form of Heaviside step function.

3.1.1. Effect of Normalized Noise Intensities $\sigma_{x}$ and $\sigma_{y}$ on Tsunami Generation Waveform. We studied the effect of the normalized noise intensities on the tsunami generation in Figures 2, 3, and 4 . It can be observed from these figures that the increase in the normalized noise intensity leads to an increase in the deformation in the bottom topography, while the wave behavior is mainly the same. Figure 6 presents a collection of the waveforms from Figure 5 at $t=t^{*}$ to get a better insight of the effect of the normalized noise intensity on the tsunami generation, where it can be observed that the increase of the normalized noise intensities on the bottom topography leads to slight difference in the peak amplitude of the waveforms in addition to an increase in oscillations in the free surface elevation. These differences can be further investigated by discussing the variance of the generated tsunami waveforms. We investigated the normalized variance $\left(\operatorname{Var} / \sigma^{2}\right)$ of the tsunami generation in Figures 7 and 8 at $h=2 \mathrm{~km}, L=100 \mathrm{~km}$, and $D=50 \mathrm{~km}$ at time $t=0.25 t^{*}, 0.5 t^{*}, 0.75 t^{*}$, and $t^{*}$. It can be seen in Figure 8 that the variance increases with the increase in time and the maximum amplitude of the variance occurs above the spreading edge of the bottom topography. The maximum variance occurs at $t=t^{*}$ (maximum amplification of the 


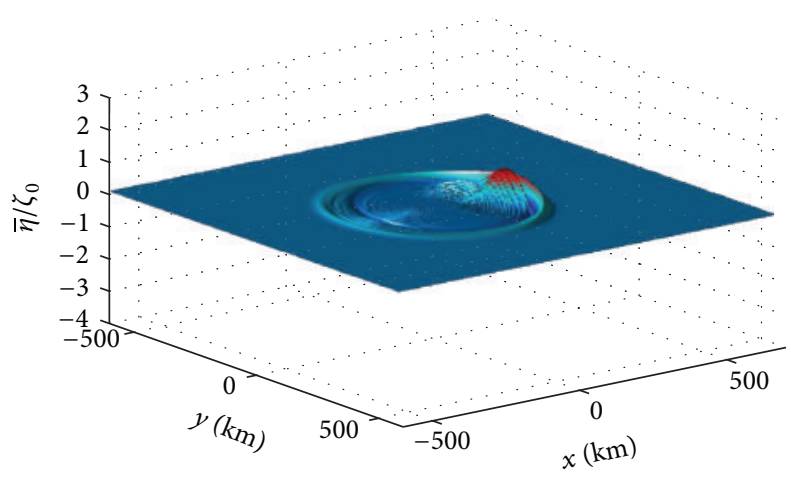

(a) For $L=50$ at $t=6 t^{*}$

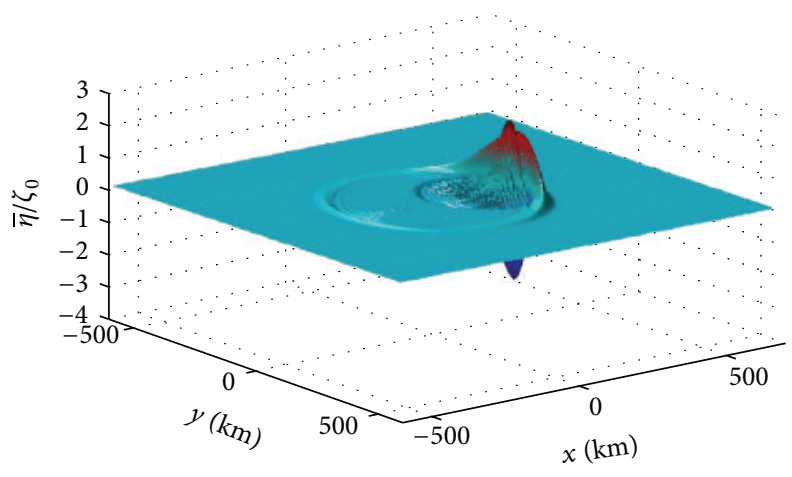

(c) For $L=150$ at $t=2 t^{*}$

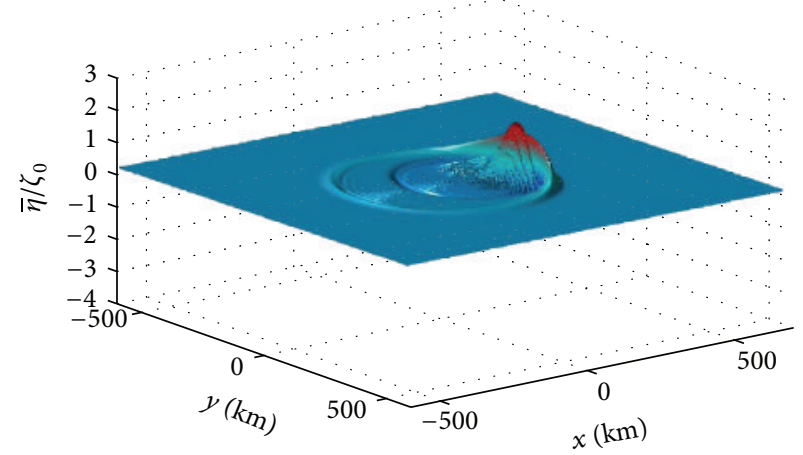

(b) For $L=100$ at $t=3 t^{*}$

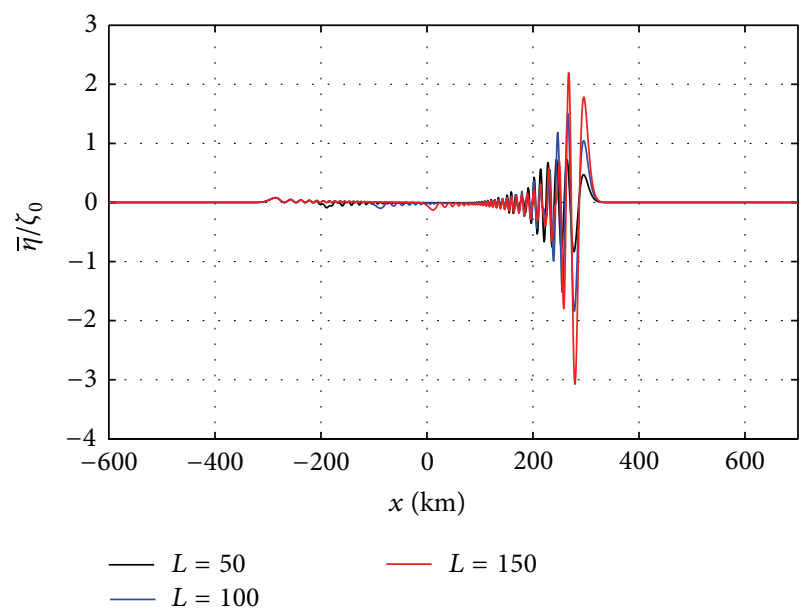

(d) Side view for $L=50,100,150$

FIGURE 19: The mean of the propagated tsunami waveforms for different propagated uplift length $L, D=50 \mathrm{~km}, h=2 \mathrm{~km}$, and $t=300 / v$, where $t^{*}=L / v$ and $v=v_{t}$.

wave). Thus we can conclude that the variance and the tsunami waveforms behave similarly during the generation process under the effect of the normalized noise intensities which is also clear in Figure 7.

3.1.2. Effect of Propagated Uplift Length L on Tsunami Generation Waveform. We illustrate the effect of the spreading uplift $L$ of the stochastic source model on the mean and the variance of the tsunami waveforms in Figures 9 and 10, respectively. It is seen from Figure 9 how the amplitude of the mean wave increases progressively as more water is lifted below the leading wave depending on its variation in time and space in the source area. The parameter that governs the amplification of the near-field water waves by focusing is the spreading length $L$. As the spreading length in the random bottom increases, the amplitude of the mean tsunami wave increases. In Figure 10, it can be observed that the maximum dispersion occurs at $L=50,100$, and $150 \mathrm{~km}$ which correspond to the maximum amplitude of the mean tsunami waves, respectively.

3.1.3. Effect of Water Depth h on Tsunami Generation Waveform. In Figures 11 and 12, we studied the variation of the water depth $h$ on the normalized mean and normalized variance of tsunami waveforms for various values of $h=2$, 4 , and $6 \mathrm{~km}$ at constant propagated uplift length $L=100 \mathrm{~km}$ and $D=50 \mathrm{~km}$ at $t=t^{*}$. It can be seen in Figures 11 and 12 that the maximum amplitude amplifications of the mean and variance of the tsunami waveforms decrease with the increases in $h$. This was obvious in Figure 11(d) where the amplitude of the normalized mean decreases from $5.173 \mathrm{~m}$ at $h=2 \mathrm{~km}$ to $2.092 \mathrm{~m}$ at $h=6 \mathrm{~km}$, while from Figure 12(d) the amplitude of the normalized variance decreases from 0.3435 at $h=2 \mathrm{~km}$ to 0.0643 at $h=6 \mathrm{~km}$. This phenomenon happens because the speed of the tsunami is related to the water depth which produces small wavelength as the velocity decreases and hence the height of the wave grows as the change of total energy of the tsunami remains constant. Mathematically, wave energy is proportional to both the length and squared height of the wave. Therefore, if the energy remains constant and the wavelength decreases, then the height must increase. The results shown in Figures 11 and 12 for the stochastic source model agree with the aspect for deterministic source models obtained by Ramadan et al. [18], Hassan et al. [17], and Hayir [40] who verified that wave amplitude is inversely proportional to water depth. 


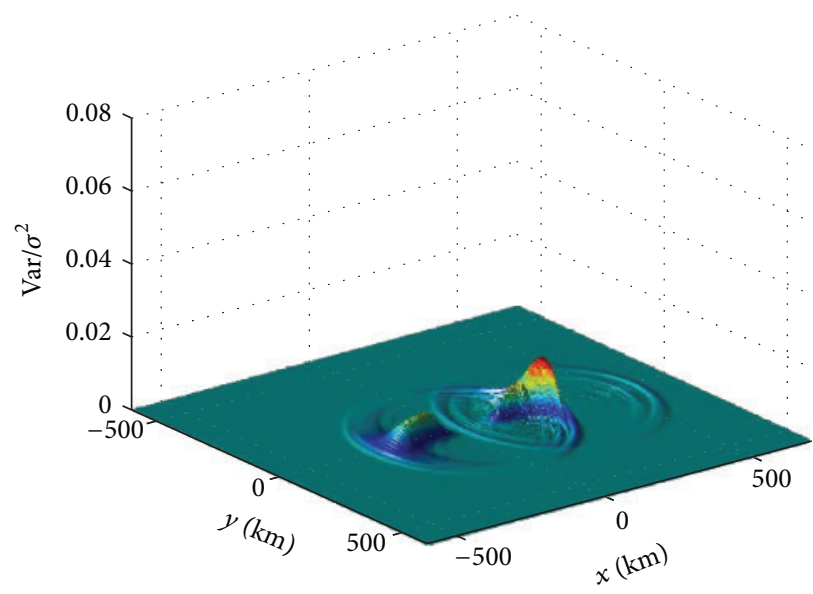

(a) For $L=50$ at $t=6 t^{*}$

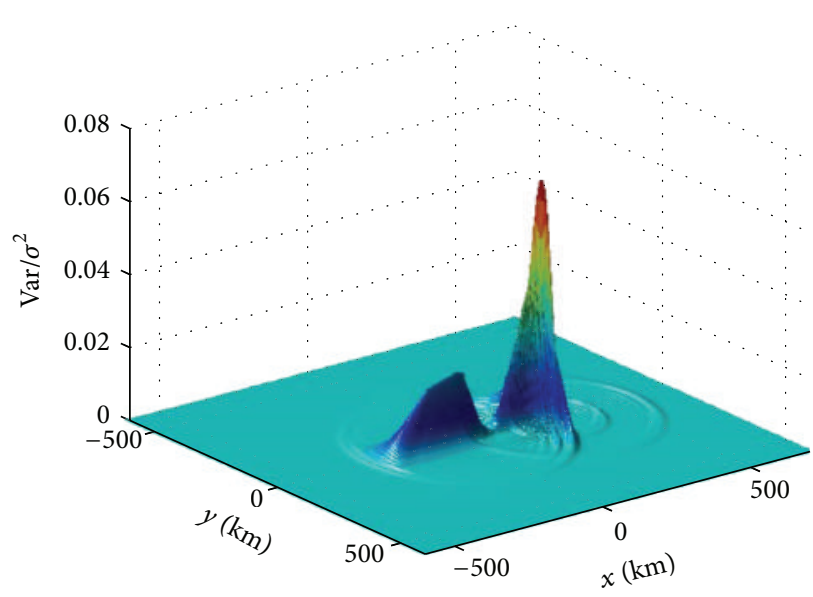

(c) For $L=150$ at $t=2 t^{*}$

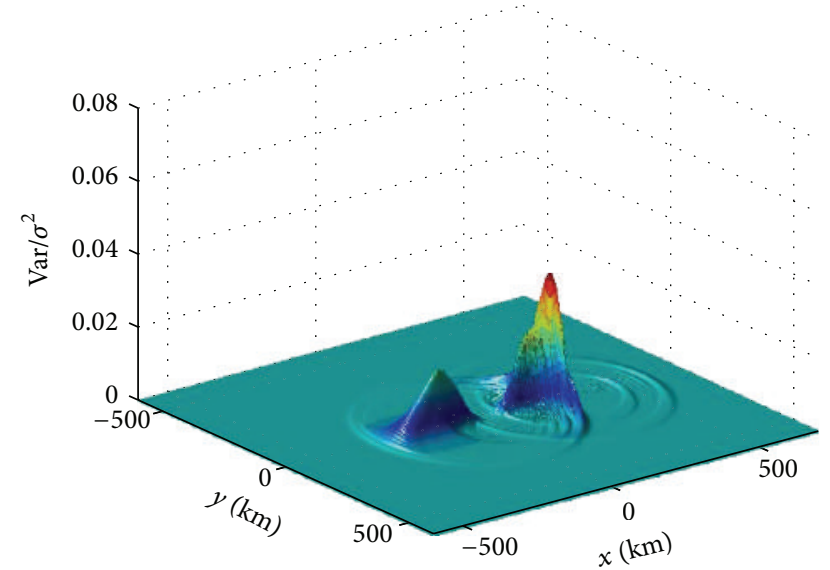

(b) For $L=100$ at $t=3 t^{*}$

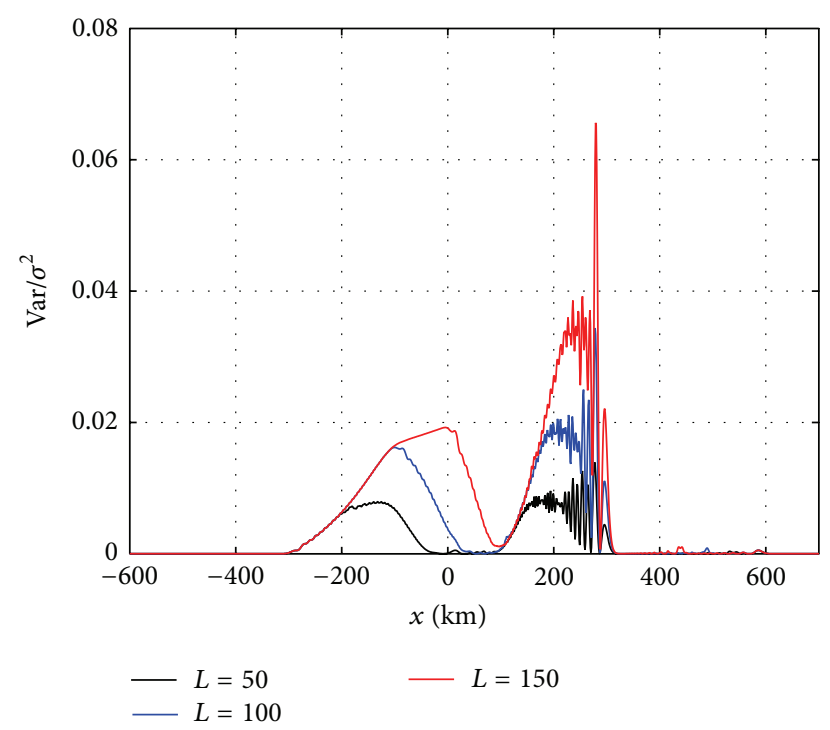

(d) Side view for $L=50,100,150$

FIGURE 20: The variance of the propagated tsunami waveforms for different propagated uplift length $L, D=50 \mathrm{~km}, h=2 \mathrm{~km}$, and $t=300 / v$, where $t^{*}=L / v$ and $v=v_{t}$.

3.2. Tsunami Propagation Evolution in Time. The effects of variations of the faulting on the propagation of tsunami wave, after the completion of the formation of the stochastic source model, are studied as a function of time $t$. These effects are studied through the investigation of the propagation of tsunami wave under the effect of the propagated uplift length $L$, water depth $h$, and the normalized noise intensities $\sigma_{x}$ and $\sigma_{y}$. Figures 13 and 14 illustrate the propagation process of the mean and the variance of tsunami propagation waveforms, respectively, in the far-field for times $t=2 t^{*}, 3 t^{*}, 4 t^{*}$, where $t^{*}=L / v$ and $v=v_{t}$. It can be seen from these figures that the maximum wave amplitude decreases with time, due to the geometric spreading and also due to dispersion, causing a train of small waves behind the main wave. It can be seen from Figure 13(d) that the amplitude of the leading normalized mean decreases from 1.774 at $t=2 t^{*}$ to 0.742 at $t=4 t^{*}$, while from Figure 14(d) the amplitude of the leading normalized variance decreases from 0.03275 at $t=2 t^{*}$ to 0.00555 at $t=4 t^{*}$.

3.2.1. Effect of Normalized Noise Intensities $\sigma_{x}$ and $\sigma_{y}$ on Tsunami Propagation Waveforms. Figures 15, 16, and 17 illustrate the propagation tsunami waveforms away from the stochastic source model presented in Figures 2(d), 3(d), and $4(\mathrm{~d})$, respectively, at noise intensities $\sigma_{x}=\sigma_{y}=\sigma=$ $0,0.2$, and 0.4 . These waveforms are the propagation of the generated tsunami waveforms represented in Figure 6 at propagated times $t=4 t^{*}, 6 t^{*}$, and $8 t^{*}$, where $t^{*}=$ $L / v$ and $v=v_{t}$. It can be seen from these figures that the maximum wave amplitude decreases during the time evolution due to geometric spreading and due to dispersion for noise intensities $\sigma_{x}=\sigma_{y}=\sigma=0,0.2$, and 0.4 . It can be observed from Figure 18 that the increase of the normalized noise intensity leads to an increase in oscillations and more 


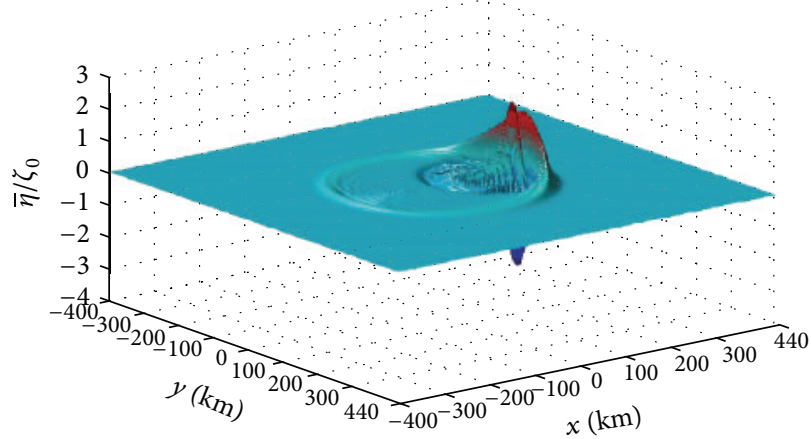

(a) At $h=2 \mathrm{~km}$

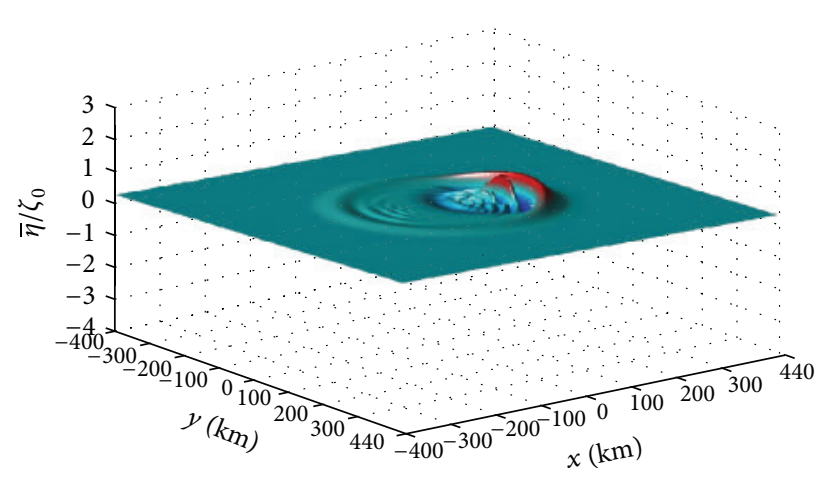

(c) At $h=6 \mathrm{~km}$

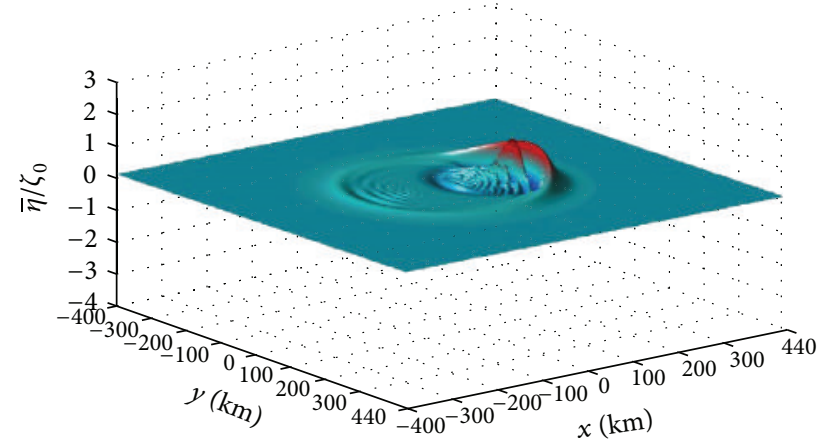

(b) At $h=4 \mathrm{~km}$

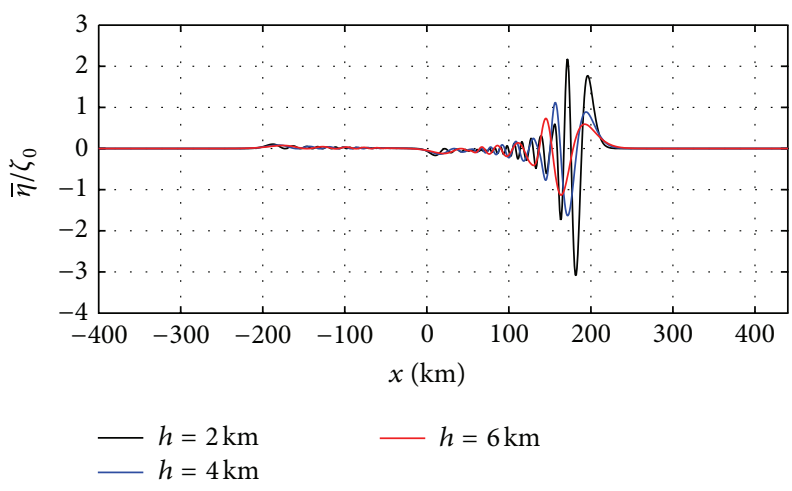

(d) Side view for $h=2,4,6 \mathrm{~km}$

FIGURE 21: Effect of the water depth $h$ on the normalized mean of the propagated tsunami waveforms at $L=100 \mathrm{~km}$ and $D=50 \mathrm{~km}$ and at $t=2 t^{*}$, where $t^{*}=L / v_{t}$ and $v=v_{t}$.

dispersion in the normalized free surface elevation but leads to a slight difference in the peak amplitude of the waveforms.

\subsubsection{Effect of Propagated Uplift Length L on Tsunami Propa-} gation Waveform. The effect of the propagated uplift length $L$ on the mean and variance of the tsunami propagation waveforms far away from the stochastic source model is illustrated in Figures 19 and 20, respectively, for spreading length $L=50,100$, and $150 \mathrm{~km}$, for times $t=6 t^{*}, 3 t^{*}$, and $2 t^{*}$, respectively, where $t^{*}=L / v$ and $v=v_{t}$. As the normalized mean wave propagates, the height of the leading wave in Figure 9(d) decreases from 3.58 at $t=t^{*}=50 / v$ to 0.4699 at $t=6 t^{*}=300 / v$ in Figure 19(d), while the wave height decreases from 5.173 at $t=t^{*}=100 / v$ to 1.045 at $t=3 t^{*}=300 / v$ and from 6.156 at $t=t^{*}=150 / v$ to 1.784 at $t=2 t^{*}=50 / v$. Similarly, the leading amplitude of the normalized variance in Figure 10(d) decreases from 0.2918 at $t=t^{*}=50 / v$ to 0.004429 at $t=6 t^{*}=300 / v$ in Figure 20(d), while the wave height decreases from 0.3435 at $t=t^{*}=100 / v$ to 0.01107 at $t=3 t^{*}=300 / v$ and from 0.3629 at $t=t^{*}=$ $150 / v$ to 0.0221 at $t=2 t^{*}=50 / v$. Hence it can be observed that the mean amplitude and variance of the wave decrease with time, due to the geometric spreading and also due to the dispersion. Moreover, the leading wave of the normalized mean and variance increases with the increase in the propagated uplift length $L$ at the same propagated time $t=300 / v$.

\subsubsection{Effect of Water Depth h on Tsunami Propagation Wave-} form. The effects of the water depth $h$ on the normalized mean and variance of the propagated tsunami waveforms are illustrated in Figures 21 and 22 for various values of $h=2,4$, and $6 \mathrm{~km}$ at constant propagated uplift length $L=100 \mathrm{~km}$, width $D=100 \mathrm{~km}$, and time $t=2 t^{*}$, where $t^{*}=L / v$ and $v=v_{t}$. It can be seen in these figures that the normalized mean and variance decrease as the water depth increases. For example, in Figure 21(d), the mean leading wave decreases from 1.775 at $h=2 \mathrm{~km}$ to 0.591 at $h=6 \mathrm{~km}$ and, in Figure 22(d), the variance decreases from 0.03275 at $h=2 \mathrm{~km}$ to 0.00366 at $h=6 \mathrm{~km}$. In addition, the oscillations of the propagated mean wave are inversely proportional to the water depth as can be seen from Figure 21(d).

Small amplitude wave theories are the linearized theories of the wave motion and the long wave theories use the numerical method of solution for the nonlinear long wave equations. We presented linearized theory to investigate the tsunami propagation waveform in the far-field; thus it can be seen from the propagated tsunami waveforms illustrated in Figures 13, 15, 16, 17, and 18 that oscillatory 


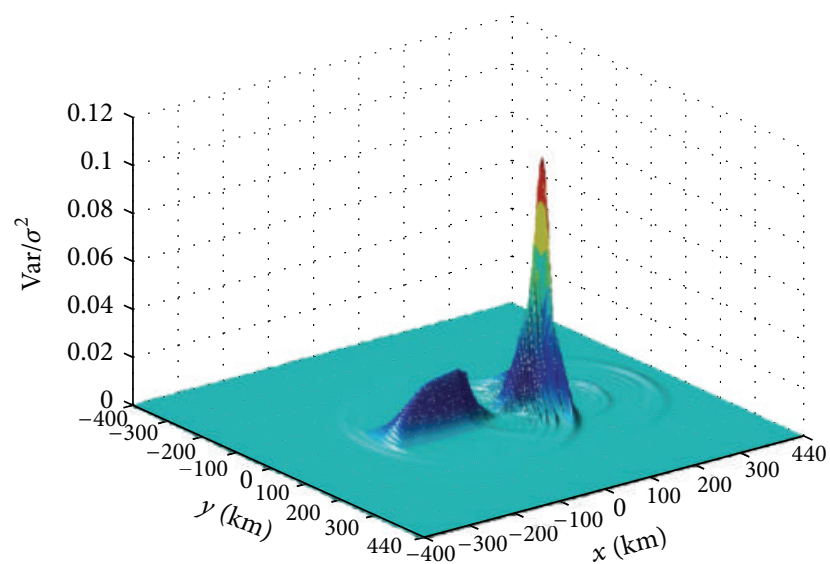

(a) At $h=2 \mathrm{~km}$

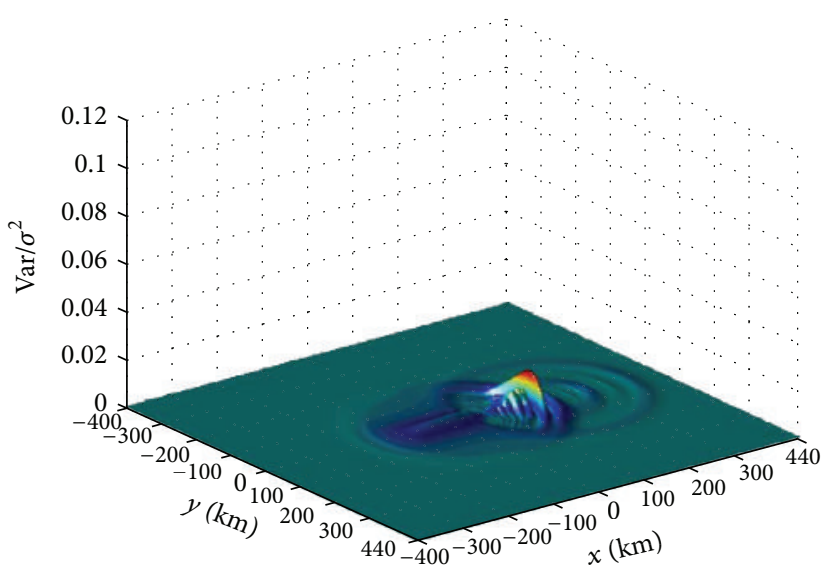

(c) At $h=6 \mathrm{~km}$

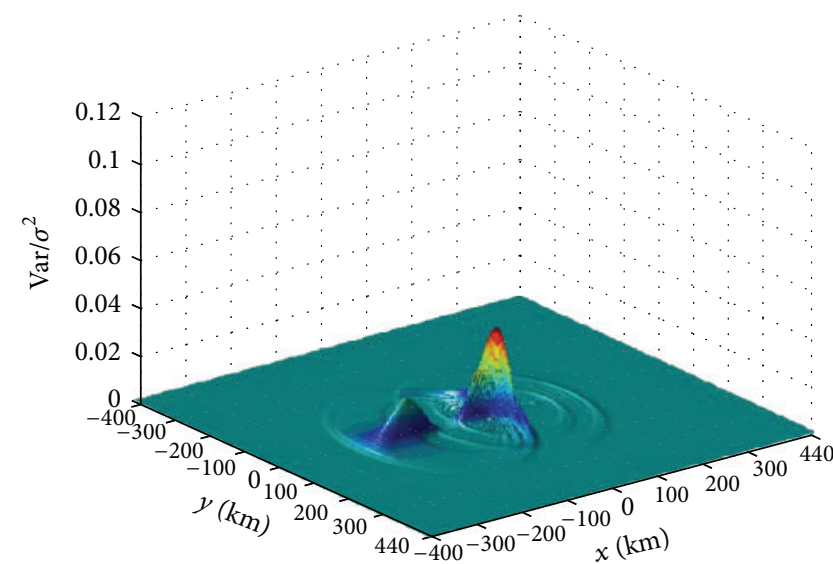

(b) At $h=4 \mathrm{~km}$

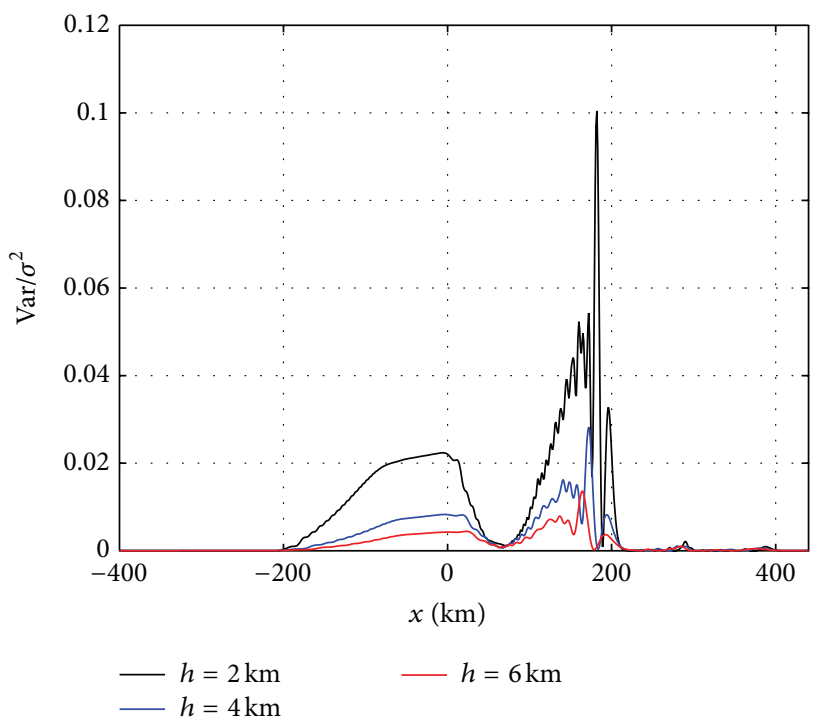

(d) Side view for $h=2,4,6 \mathrm{~km}$

FiguRE 22: Effect of the water depth $h$ on the normalized variance of the propagated tsunami waveforms at $L=100 \mathrm{~km}$ and $D=50 \mathrm{~km}$ and at $t=2 t^{*}$, where $t^{*}=L / v_{t}$ and $v=v_{t}$.

wave train develops in the far-field during propagation and the amplitude of the leading wave decays and no waves of permanent form evolve; see [9].

\section{Conclusion}

In this paper, the process of tsunami generation and propagation was investigated over a stochastic bottom topography represented by a sliding Heaviside step function under the influence of two independent Gaussian white noise processes in the $x$ - and $y$-directions, respectively. We demonstrated the waveform amplification resulting from stochastic source spreading and wave focusing and the tsunami propagation in the far-field. Due to the stochastic nature of the bottom topography, the free surface elevation becomes a stochastic process having infinite number of trajectories. Therefore we derived mathematically the mean and variance of the stochastic tsunami waveforms and illustrated them graphically to get a better insight of the overall behavior of the stochastic tsunami waveforms under the effect of the spreading length, noise intensities, and the water depth. It was observed that, near the source, the wave has large amplitude as the wave builds up progressively as $t$ increases and the focusing and the amplification of tsunami amplitudes occur above the spreading edge of the stochastic source model, while as the tsunami further departed away from the source the amplitude decreased due to dispersion. Moreover, the mean amplitude and the variance were found to be proportional to the propagation length of the stochastic source model and inversely proportional to the water depth. In addition, the maximum dispersion (variance) was found to occur at the corresponding maximum or minimum amplitude of mean generated wave. Also, when increasing the normalized noise intensity, the oscillations of the free surface elevation increase. In addition to an increase in the difference in the peak amplitudes of the waveforms, especially the leading 
wave, during the propagation process, it was observed that the increase in the noise intensity shows more dispersion and oscillation in the propagated free surface elevation.

\section{Conflict of Interests}

The authors declare that there is no conflict of interests regarding the publication of this paper.

\section{References}

[1] M. S. Abou-Dina and F. M. Hassan, "Generation and propagation of nonlinear tsunamis in shallow water by a moving topography," Applied Mathematics and Computation, vol. 177, no. 2, pp. 785-806, 2006.

[2] F. M. Hassan, "Boundary integral method applied to the propagation of non-linear gravity waves generated by a moving bottom," Applied Mathematical Modelling, vol. 33, no. 1, pp. 451466, 2009.

[3] N. Zahibo, E. Pelinovsky, T. Talipova, A. Kozelkov, and A. Kurkin, "Analytical and numerical study of nonlinear effects at tsunami modeling," Applied Mathematics and Computation, vol. 174, no. 2, pp. 795-809, 2006.

[4] H. S. Hassan, K. T. Ramadan, and S. N. Hanna, "Numerical solution of the rotating shallow water ows with topography using the fractional steps method," Applied Mathematics, vol. 1, no. 2, pp. 104-117, 2010.

[5] V. V. Titov and C. E. Synolakis, "Modeling of breaking and nonbreaking long-wave evolution and runup using VTCS-2," Journal of Waterway, Port, Coastal \& Ocean Engineering, vol. 121, no. 6, pp. 308-316, 1995.

[6] B. Gurevich, A. Jeffrey, and E. N. Pelinovsky, "A method for obtaining evolution equations for nonlinear waves in a random medium," Wave Motion, vol. 17, no. 3, pp. 287-295, 1993.

[7] A. de Bouard, W. Craig, O. Díaz-Espinosa, P. Guyenne, and C. Sulem, "Long wave expansions for water waves over random topography," Nonlinearity, vol. 21, no. 9, pp. 2143-2178, 2008.

[8] A. Nachbin, "Discrete and continuous random water wave dynamics," Discrete and Continuous Dynamical Systems A, vol. 28, no. 4, pp. 1603-1633, 2010.

[9] J. L. Hammack, "A note on tsunamis: their generation and propagation in an ocean of uniform depth," Journal of Fluid Mechanics, vol. 60, pp. 769-799, 1973.

[10] M. I. Todorovska and M. D. Trifunac, "Generation of tsunamis by a slowy spreading uplift of the sea floor," Soil Dynamics and Earthquake Engineering, vol. 21, no. 2, pp. 151-167, 2001.

[11] M. I. Todorovska, A. Hayir, and M. D. Trifunac, "A note on tsunami amplitudes above submarine slides and slumps," Soil Dynamics and Earthquake Engineering, vol. 22, no. 2, pp. 129$141,2002$.

[12] M. D. Trifunac, A. Hayir, and M. I. Todorovska, "A note on the effects of nonuniform spreading velocity of submarine slumps and slides on the near-field tsunami amplitudes," Soil Dynamics and Earthquake Engineering, vol. 22, no. 3, pp. 167-180, 2002.

[13] A. Hayir, "The effects of variable speeds of a submarine block slide on near-field tsunami amplitudes," Ocean Engineering, vol. 30, no. 18, pp. 2329-2342, 2003.

[14] D. Dutykh, F. Dias, and Y. Kervella, "Linear theory of wave generation by a moving bottom," Comptes Rendus Mathématique. Académie des Sciences. Paris, vol. 343, no. 7, pp. 499-504, 2006.
[15] D. Dutykh and F. Dias, "Water waves generated by a moving bottom," in Tsunami and Nonlinear Waves, pp. 65-95, Springer, Berlin, Germany, 2007.

[16] Y. Kervella, D. Dutykh, and F. Dias, "Comparison between three-dimensional linear and nonlinear tsunami generation models," Theoretical and Computational Fluid Dynamics, vol. 21, no. 4, pp. 245-269, 2007.

[17] H. S. Hassan, K. T. Ramadan, and S. N. Hanna, "Generation and propagation of tsunami by a moving realistic curvilinear slide shape with variable velocities in linearized shallow-water wave theory," Engineering, vol. 2, no. 7, pp. 529-549, 2010.

[18] K. T. Ramadan, H. S. Hassan, and S. N. Hanna, "Modeling of tsunami generation and propagation by a spreading curvilinear seismic faulting in linearized shallow-water wave theory," Applied Mathematical Modelling, vol. 35, no. 1, pp. 61-79, 2011.

[19] K. T. Ramadan, M. A. Omar, and A. A. Allam, "Modeling of tsunami generation and propagation by a spreading seismic faulting in two orthogonal directions in linearized shallowwater wave theory," 2012, http://etms-eg.org/.

[20] R. L. Wiegel, Earthquake Engineering, Prentice-Hall, Englewood Cliffs, NJ, USA, 1970.

[21] O. Rascón and A. G. Villarreal, "On a stochastic model to estimate tsunami risk," Journal of Hydraulic Research, vol. 13, no. 4, pp. 383-403, 1975.

[22] S. Nakamura, "Estimate of exceedance probability of tsunami occurrence in the eastern pacific," Marine Geodesy, vol. 10, no. 2, pp. 195-209, 1986.

[23] E. L. Geist, "Complex earthquake rupture and local tsunamis," Journal of Geophysical Research B, vol. 107, no. 5, pp. 2086-2100, 2002.

[24] E. L. Geist, "Rapid tsunami models and earthquake source parameters: far-field and local applications," ISET Journal of Earthquake Technology, vol. 42, no. 4, pp. 127-136, 2005.

[25] W. Craig, P. Guyenne, and C. Sulem, "Water waves over a random bottom," Journal of Fluid Mechanics, vol. 640, pp. 79$107,2009$.

[26] H. Manouzi and M. Seaïd, "Solving Wick-stochastic water waves using a Galerkin finite element method," Mathematics and Computers in Simulation, vol. 79, no. 12, pp. 3523-3533, 2009.

[27] D. Dutykh, C. Labart, and D. Mitsotakis, "Long wave run-up on random beaches," Physical Review Letters, vol. 107, no. 18, Article ID 184504, 2011.

[28] M. A. Omar, A. A. Allam, and K. T. Ramadan, "Generation and propagation of tsunami wave under the effect of stochastic bottom topography," 2012, http://etms-eg.org/.

[29] K. T. Ramadan, M. A. Omar, and A. A. Allam, "Modeling of tsunami generation and propagation under the effect of stochastic submarine landslides and slumps spreading in two orthogonal directions," Ocean Engineering, vol. 75, pp. 90-111, 2014.

[30] F. C. Klebaner, Introduction to Stochastic Calculus with Applications, Imperial College Press, London, UK, 2nd edition, 2005.

[31] P. E. Kloeden and E. Platen, Numerical Solution of Stochastic Differential Equations, Springer, Berlin, Germany, 1992.

[32] B. Øksendal, Stochastic Differential Equations: An Introduction with Applications, Springer, Berlin, Germany, 1995.

[33] M. A. Omar, A. Aboul-Hassan, and S. I. Rabia, "The composite Milstein methods for the numerical solution of Stratonovich stochastic differential equations," Applied Mathematics and Computation, vol. 215, no. 2, pp. 727-745, 2009. 
[34] M. A. Omar, A. Aboul-Hassan, and S. I. Rabia, "The composite Milstein methods for the numerical solution of Ito stochastic differential equations," Journal of Computational and Applied Mathematics, vol. 235, no. 8, pp. 2277-2299, 2011.

[35] H. H. Sherief, N. M. El-Maghraby, and A. A. Allam, "Stochastic thermal shock problem in generalized thermoelasticity," Applied Mathematical Modelling, vol. 37, no. 3, pp. 762-775, 2013.

[36] H. Kanamori and G. S. Stewart, "A slowly earthquake," Physics of the Earth and Planetary Interiors, vol. 18, pp. 167-175, 1972.

[37] P. G. Silver and T. H. Jordan, "Total-moment spectra of fourteen large earthquakes," Journal of Geophysical Research, vol. 88, no. 4, pp. 3273-3293, 1983.

[38] M. D. Trifunac and M. I. Todorovska, "A note on differences in tsunami source parameters for submarine slides and earthquakes," Soil Dynamics and Earthquake Engineering, vol. 22, no. 2, pp. 143-155, 2002.

[39] M. D. Trifunac, A. Hayir, and M. I. Todorovska, "Was grand banks event of 1929 a slump spreading in two directions?" Soil Dynamics and Earthquake Engineering, vol. 22, no. 5, pp. 349360, 2002.

[40] A. Hayir, "Ocean depth effects on tsunami amplitudes used in source models in linearized shallow-water wave theory," Ocean Engineering, vol. 31, no. 3-4, pp. 353-361, 2004. 


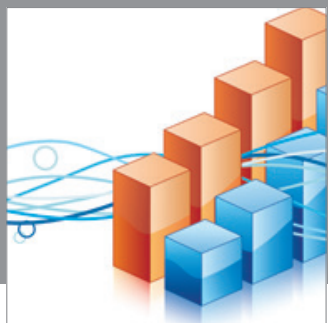

Advances in

Operations Research

mansans

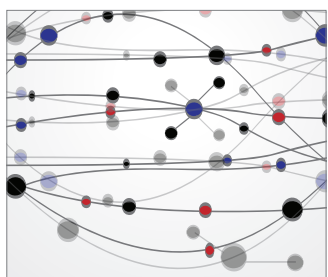

The Scientific World Journal
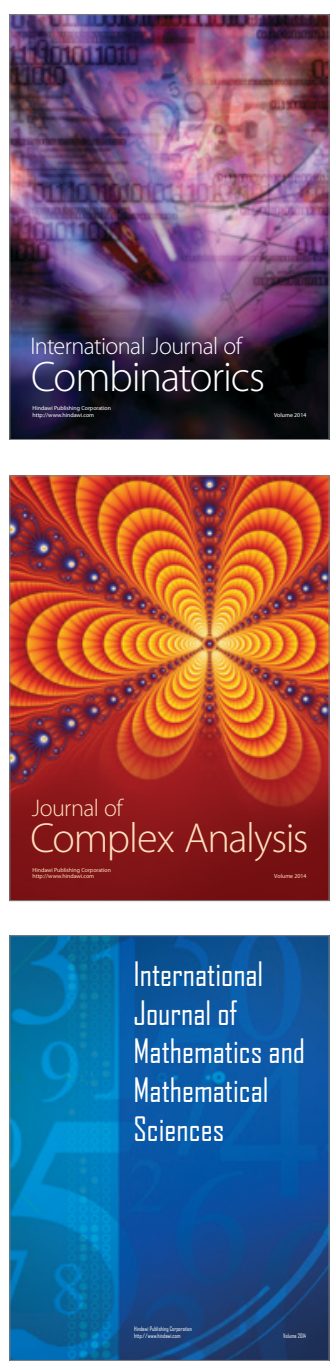
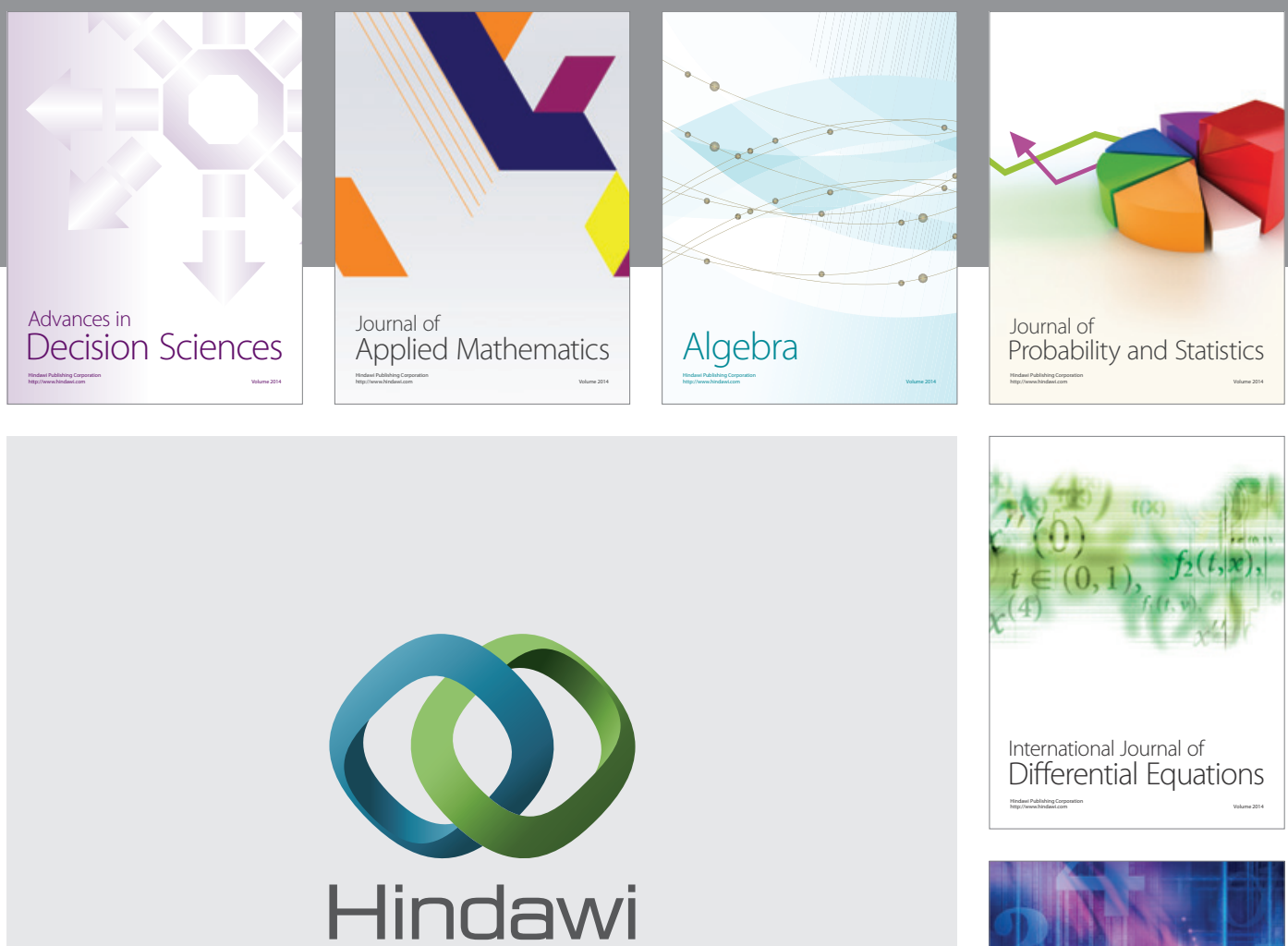

Submit your manuscripts at http://www.hindawi.com
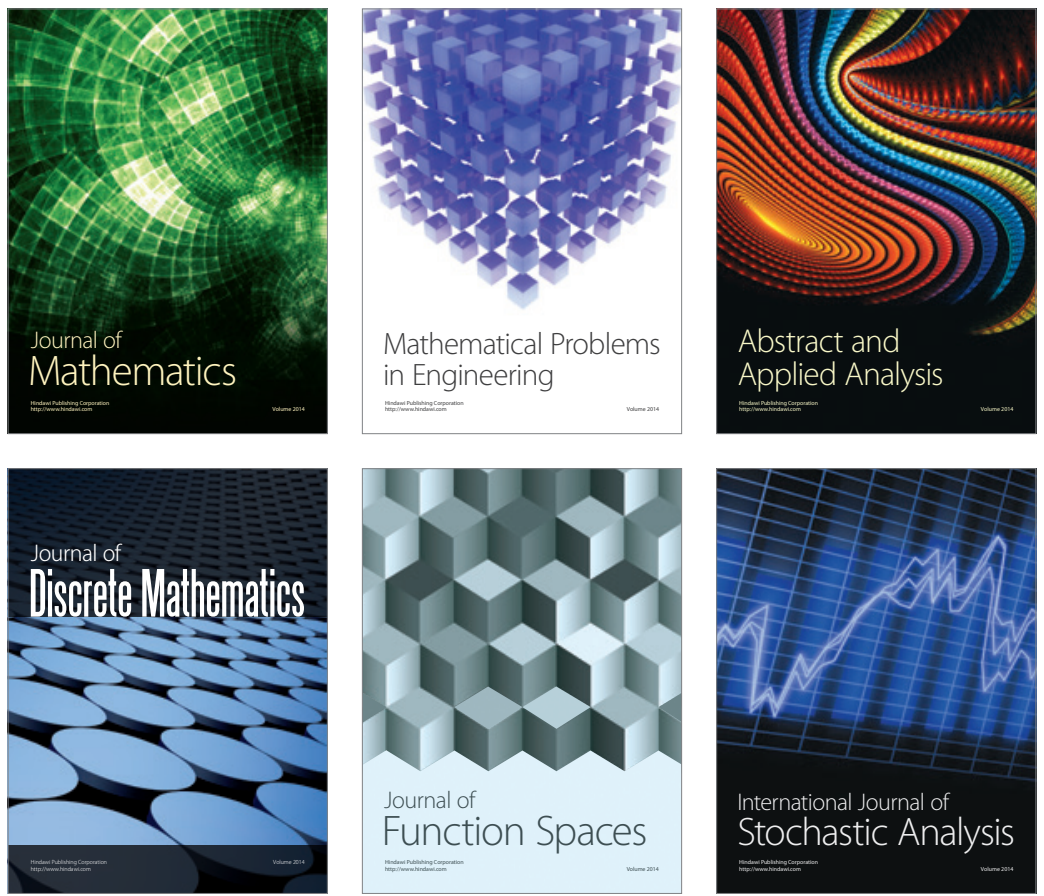

Journal of

Function Spaces

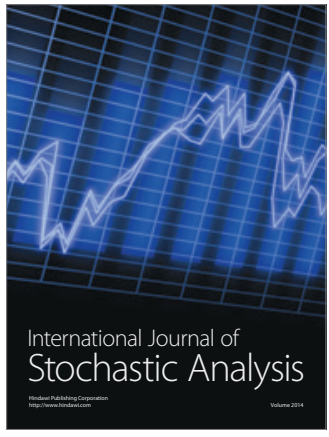

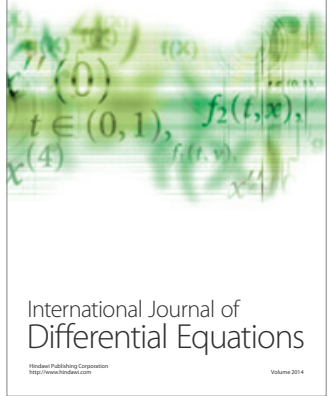
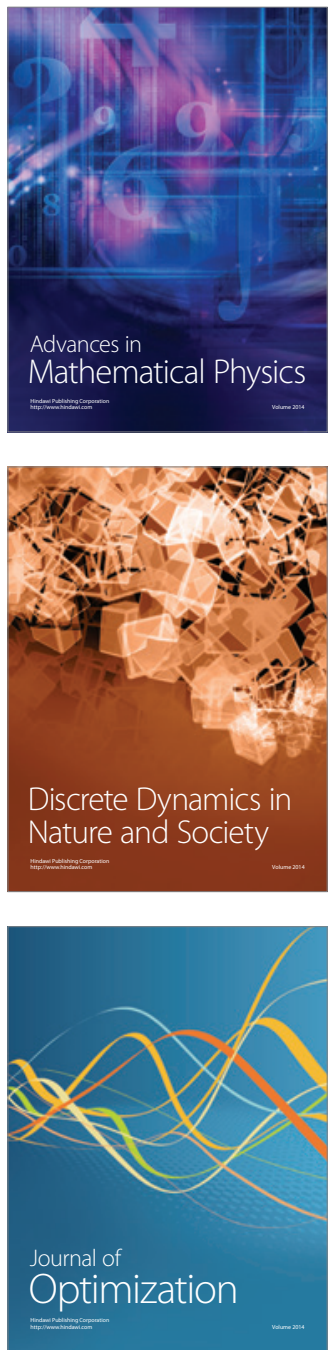